\title{
ASYMPTOTICS FOR THE PARTIAL AUTOCORRELATION FUNCTION OF A STATIONARY PROCESS
}

\author{
AKIHIKO INOUE
}

\section{INTRODUCTION}

The purpose of this paper is to study the long-time behaviour of the partial autocorrelation function of a stationary process.

Let $\left\{X_{n}\right\}=\left\{X_{n}: n \in \mathbb{Z}\right\}$ be a real, zero-mean, weakly stationary process, defined on a probability space $(\Omega, \mathcal{F}, P)$, which we shall simply call a stationary process. Throughout this paper, we assume that $\left\{X_{n}\right\}$ is purely nondeterministic (see $\S 2$ ). The autocovariance function $\gamma(\cdot)$ of $\left\{X_{n}\right\}$ is defined by

$$
\gamma(n):=E\left[X_{n} X_{0}\right] \quad(n \in \mathbb{Z})
$$

We denote by $H$ the closed real linear hull of $\left\{X_{k}: k \in \mathbb{Z}\right\}$ in $L^{2}(\Omega, \mathcal{F}, P)$. Then $H$ is a real Hilbert space with inner product

$$
\left(Y_{1}, Y_{2}\right):=E\left[Y_{1} Y_{2}\right]
$$

and norm

$$
\|Y\|:=(Y, Y)^{1 / 2}
$$

For $n \geq 1$, we write $H_{[1, n]}$ for the subspace of $H$ spanned by $\left\{X_{1}, \ldots, X_{n}\right\}$, and $P_{[1, n]}$ for the orthogonal projection operator of $H$ onto $H_{[1, n]}$.

The partial autocorrelation $\alpha(n)$ is the correlation coefficient of the two residuals obtained after regressing $X_{0}$ and $X_{n}$ on the intermediate observations $X_{1}$, $\ldots, X_{n-1}$. More precisely, the partial autocorrelation function $\alpha(\cdot)$ of $\left\{X_{n}\right\}$ is defined by

$$
\alpha(n):=\frac{E\left[Z_{n}^{+} Z_{n}^{-}\right]}{E\left[\left(Z_{n}^{+}\right)^{2}\right]^{1 / 2} \cdot E\left[\left(Z_{n}^{-}\right)^{2}\right]^{1 / 2}} \quad(n=2,3, \ldots),
$$

where

$$
Z_{n}^{+}:=X_{n}-P_{[1, n-1]} X_{n}, \quad Z_{n}^{-}:=X_{0}-P_{[1, n-1]} X_{0}
$$


Furthermore, $\alpha(1)$ is defined by

$$
\alpha(1):=\gamma(1) / \gamma(0) .
$$

We think of $Z_{n}^{+}$as the part of $X_{0}$ that cannot be explained by the intermediate observations $X_{1}, \ldots, X_{n-1}$, and $Z_{n}^{-}$as the part of $X_{n}$ that cannot be explained by these observations. So the partial autocorrelation $\alpha(n)$ is a kind of 'pure' correlation coefficient between $X_{0}$ and $X_{n}$. See Brockwell-Davis [BD, $\S 3.4$ and $\S 5.2]$ for background.

One of the important facts about the partial autocorrelation function $\alpha(\cdot)$ is that we can calculate the value of $\alpha(n)$ easily (at least numerically) from the values of $\gamma(0), \gamma(1), \ldots, \gamma(n)$. To do that, one may just use the Durbin-Levinson algorithm (see [BD, Proposition 5.2.1]). Moreover, if we look at the algorithm carefully, we find that conversely the values of $\gamma(0), \alpha(1), \ldots, \alpha(n)$ determine the value of $\gamma(n)$. In this sense, the partial autocorrelation function $\alpha(\cdot)$ has the same information as the autocovariance function $\gamma(\cdot)$.

What does $\alpha(n)$ look like for $n$ large? This seemingly simple problem, which is our central concern in this paper, turns out to be much harder than it looks at first. The difficulty is related to the fact that the definition of partial autocorrelation function involves the prediction from a finite part of time. This setting makes the asymptotic analysis particularly difficult.

We are especially interested in the case in which $\left\{X_{n}\right\}$ is a long-memory process; roughly speaking, this means that the autocovariance $\gamma(k)$ of $\left\{X_{n}\right\}$ tends to zero as $k \rightarrow \infty$ so slowly that $\gamma(\cdot)$ is not summable (see [BD, §13.2]). In our main theorem (Theorem 2.1), we determine the desired asymptotics for the partial autocorrelation function, modulo absolute value, for a class of stationary processes which includes long-memory processes. Our result presents a surprising regularity in the asymptotics. More precisely, let $-\infty<d<\frac{1}{2}$ and $\ell$ be a slowly varying function at infinity (see §2). Then under certain conditions (on the $\operatorname{MA}(\infty)$ and $\operatorname{AR}(\infty)$ coefficients of $\left.\left\{X_{n}\right\}\right)$, it is shown that,

$$
\gamma(n) \sim n^{2 d-1} \ell(n) \quad(n \rightarrow \infty)
$$


implies

$$
|\alpha(n)| \sim \frac{\gamma(n)}{\sum_{k=-n}^{n} \gamma(k)} \quad(n \rightarrow \infty) .
$$

In particular, if $0<d<\frac{1}{2}$, that is, $0<1-2 d<1$, then

$$
|\alpha(n)| \sim \frac{d}{n} \quad(n \rightarrow \infty) .
$$

We wish to emphasize some features of the results presented just above. It should be noted that the assumption (1.1) simply says $\gamma(\cdot)$ is regularly varying with negative index (cf. Bingham et al. [BGT, §1.4.2]) since the index $2 d-1$ may take any negative values. It is perhaps surprising that there exists such a simple formula as (1.2). As one sees, the result (1.3) for the long-memory case $0<d<\frac{1}{2}$ is particularly simple; the index over $n$ is one, whence independent of $d$, and the slowly varying function $\ell$ has even disappeared. We also notice that the quantity $d$, which is important in a long-memory process, appears explicitly in $(1.3)$.

We tackle the problem above via the asymptotic analysis of the relevant expected prediction error (Theorems 6.4,6.6 and 6.7). The idea is to use the precise asymptotics for the sequence $\left\{c_{n}\right\}$ of $\mathrm{MA}(\infty)$ coefficients and the sequence $\left\{a_{n}\right\}$ of $\operatorname{AR}(\infty)$ coefficients. Here we note that the sequences $\left\{c_{n}\right\}$ and $\left\{a_{n}\right\}$ are defined for every purely nondeterministic stationary process $(\S 2)$. To deduce the desired asymptotic behaviour of the partial autocorrelation function from that of the prediction error, we use a Tauberian argument. So naturally we need an adequate Tauberian condition. It turns out that the most elementary Tauberian condition, that is, monotonicity, is available here.

We verify the desired monotonicity by an explicit representation of the prediction error in terms of $\left\{c_{n}\right\}$ and $\left\{a_{n}\right\}$ (Theorems 4.5 and 4.6). This representation, in turn, is obtained by an argument on the geometry of the Hilbert space $H$ (Theorem 4.1). Here we use a discrete-time analogue of the Seghier-Dym theorem. The (original) Seghier-Dym theorem ([S], [Dy2]) concerns the intersection of past and future of a continuous-time stationary process. This theorem originates in the work of Levinson-McKean [LM]. We prove an analogue of this theorem for discrete-time stationary processes (Theorem 3.1) and then apply it to our problem. 
In the main theorem, we assume some conditions which are given in terms of $\left\{c_{n}\right\}$ and $\left\{a_{n}\right\}$. As an example, we consider the stationary processes whose autocovariance functions are completely monotone. This property for a stationary process is called reflection positivity. For example, if $-\infty<d<\frac{1}{2}$, then the stationary process with autocovariance function of the form $\gamma(n)=(1+|n|)^{2 d-1}$ has reflection positivity (Example in $§ 7$ ). See Okabe [O] as well as [I2, OI] for earlier work. Since we wish to consider long-memory processes (as well as short-memory ones), our class of stationary processes with reflection positivity is different from those studied in these references; the latter do not include long-memory processes. We show that the stationary processes in our class satisfy the conditions of the main theorem (Theorem 7.3).

We state the main theorem in $\S 2$. In $\S 3$, we prove the Seghier-Dym type theorem. In $\S 4$, we give some representation theorems in terms of $\left\{c_{n}\right\}$ and $\left\{a_{n}\right\}$. We obtain the necessary asymptotics for $\left\{c_{n}\right\}$ and $\left\{a_{n}\right\}$ in $\S 5$. In $\S 6$, we first show the necessary results on the asymptotics for the prediction error and then prove the main theorem using them. In $\S 7$, we consider the stationary processes with reflection positivity and show that they satisfy the conditions of the main theorem.

\section{MAIN THEOREM}

In this section, we shall state the main theorem. To do that, we need some notation.

Let $\left\{X_{n}\right\}=\left\{X_{n}: n \in \mathbb{Z}\right\}$ be a stationary process; as stated in $\S 1$, this means that $\left\{X_{n}\right\}$ is a real, zero-mean, weakly stationary process, defined on a probability space $(\Omega, \mathcal{F}, P)$. Let $\gamma(\cdot)$ be the autocovariance function of $\left\{X_{n}\right\}$. As we also stated in $\S 1$, we write $H$ for the real Hilbert space spanned by $\left\{X_{k}\right.$ : $k \in \mathbb{Z}\}$ in $L^{2}(\Omega, \mathcal{F}, P)$, with inner product $\left(Y_{1}, Y_{2}\right):=E\left[Y_{1} Y_{2}\right]$ and norm $\|Y\|:=$ $(Y, Y)^{1 / 2}$. For $I \subset \mathbb{Z}$, denote by $H_{I}$ the closed real linear hull of $\left\{X_{k}: k \in I\right\}$ in $H$. In particular, for $m \in \mathbb{Z}$ and $n \in \mathbb{Z}$ with $m \leq n$, we write $H_{(-\infty, m]}, H_{[m, \infty)}$ and $H_{[m, n]}$ for $H_{I}$ with $I=\{k \in \mathbb{Z}:-\infty<k \leq m\},\{k \in \mathbb{Z}: m \leq k<\infty\}$ and $\{k \in \mathbb{Z}: m \leq k \leq n\}$, respectively. For $I \subset \mathbb{Z}$, we denote by $P_{I}$ the orthogonal 
projection operator of $H$ onto $H_{I}$. We write $P_{I}^{\perp}:=I_{H}-P_{I}$, where $I_{H}$ is the identity map of $H$. So $P_{I}^{\perp}$ is the orthogonal projection operator of $H$ onto $H_{I}^{\perp}$.

As we stated in $§ 1$, we assme throughout this paper that the stationary process $\left\{X_{n}\right\}$ is purely nondeterministic, that is,

$$
\bigcap_{n=-\infty}^{\infty} H_{(-\infty, n]}=\{0\}
$$

or, equivalently, there exists a positive even and integrable function $\Delta(\cdot)$ on $(-\pi, \pi)$ such that

$$
\gamma(n)=\int_{-\pi}^{\pi} e^{i n \theta} \Delta(\theta) d \theta \quad(n \in \mathbb{Z}), \quad \int_{-\pi}^{\pi}|\log \Delta(\theta)| d \theta<\infty
$$

(see [BD, §5.7] and Rozanov [Ro, Chapter II]; in the latter, the term linearly regular is used instead of purely nondeterministic). We call $\Delta(\cdot)$ the spectral density of $\left\{X_{n}\right\}$. It should be pointed out that there exists an a.e. ambiguity for $\Delta(\cdot)$. We define the outer function $h(\cdot)$ of $\left\{X_{n}\right\}$ by

$$
h(z):=(2 \pi)^{1 / 2} \exp \left\{\frac{1}{4 \pi} \int_{-\pi}^{\pi} \frac{e^{i \theta}+z}{e^{i \theta}-z} \log \Delta(\theta) d \theta\right\} \quad(z \in \mathbb{C},|z|<1) .
$$

The function $h(\cdot)$ is actually an outer function which is in the Hardy space $H^{2+}$ of class 2 over the unit disk $|z|<1$ (see Rudin [Ru, Definition 17.14]).

Let $c_{n}$ be the power series coefficients of $h(z)$ :

$$
h(z)=\sum_{n=0}^{\infty} c_{n} z^{n} \quad(|z|<1) .
$$

The coefficients $c_{n}$ are real and satisfy $\sum_{0}^{\infty}\left(c_{n}\right)^{2}<\infty$ (see $[\mathrm{Ru}$, Theorem 17.12]). We call $c_{n}$ the $n$th $\mathrm{MA}(\infty)$ coefficient of $\left\{X_{n}\right\}$ (see (4.7) below for background). The sequence $\left\{c_{n}\right\}$ is often called the canonical representation kernel of $\left\{X_{n}\right\}$, too. Now the outer function $h(z)$ has no zeros in $|z|<1$, whence we have another holomorphic function $1 / h(z)$ in $|z|<1$. Let $a_{n}$ be the power series coefficients of the function $-1 / h(z)$ :

$$
-\frac{1}{h(z)}=\sum_{n=0}^{\infty} a_{n} z^{n} \quad(|z|<1)
$$

Then $a_{n}$ are also real. We call $a_{n}$ the $n$th $\operatorname{AR}(\infty)$ coefficient of $\left\{X_{n}\right\}$ (see (4.9) below for background). Since

$$
\left(\sum_{n=0}^{\infty} a_{n} z^{n}\right)\left(\sum_{n=0}^{\infty} c_{n} z^{n}\right)=-1 \quad(|z|<1)
$$


we have the following relation between $\left\{c_{n}\right\}$ and $\left\{a_{n}\right\}$ :

$$
\sum_{j=0}^{n} a_{j} c_{n-j}=-\delta_{n 0} \quad(n \geq 0) .
$$

We state the main theorem under the following conditions on the sequences $\left\{c_{n}\right\}_{n=0}^{\infty},\left\{a_{n}\right\}_{n=0}^{\infty}$ and $\left\{a_{n}-a_{n+1}\right\}_{n=0}^{\infty}$ :

$$
\begin{gathered}
c_{n} \geq 0 \text { for all } n \geq 0 ; \\
\left\{c_{n}\right\} \text { is eventually decreasing to zero; } \\
\left\{a_{n}\right\} \text { is eventually decreasing to zero; } \\
\left\{a_{n}-a_{n+1}\right\} \text { is eventually decreasing to zero. }
\end{gathered}
$$

We write $\mathcal{R}_{0}$ for the class of slowly varying functions at infinity: the class of positive, measurable $\ell$, defined on some neighbourhood $[A, \infty)$ of infinity, such that

$$
\lim _{x \rightarrow \infty} \ell(\lambda x) / \ell(x)=1 \quad \text { for all } \lambda>0
$$

(see [BGT, Chapter 1] for background). Let $\ell \in \mathcal{R}_{0}$, and choose $B$ so large that $\ell(\cdot)$ is locally bounded on $[B, \infty)$ (see [BGT, Corollary 1.4.2]). When we say $\int^{\infty} \ell(s) d s / s=\infty$, it means that $\int_{B}^{\infty} \ell(s) d s / s=\infty$. If so, then we define another slowly varying function $\tilde{\ell}$ by

$$
\tilde{\ell}(x):=\int_{B}^{x} \frac{\ell(s)}{s} d s \quad(x \geq B)
$$

(see [BGT, $\S 1.5 .6]$ ). The asymptotic behaviour of $\tilde{\ell}(x)$ as $x \rightarrow \infty$ does not depend on the choice of $B$ because we have assumed that $\int^{\infty} \ell(s) d s / s=\infty$.

Let $\alpha(\cdot)$ be the partial autocorrelation function of $\left\{X_{n}\right\}$. Here is the main theorem.

Theorem 2.1. Let $-\infty<d<\frac{1}{2}$ and $\ell \in \mathcal{R}_{0}$. We assume (C1), (C2), (A1), and (A2). Suppose that

$$
\gamma(n) \sim n^{2 d-1} \ell(n) \quad(n \rightarrow \infty) .
$$

Then

$$
|\alpha(n)| \sim \frac{\gamma(n)}{\sum_{k=-n}^{n} \gamma(k)} \quad(n \rightarrow \infty)
$$

holds. In other words, 
(1) if $0<d<\frac{1}{2}$, then

$$
|\alpha(n)| \sim \frac{d}{n} \quad(n \rightarrow \infty)
$$

(2) if $d=0$ and $\int^{\infty} \ell(s) d s / s=\infty$, then

$$
|\alpha(n)| \sim n^{-1} \frac{\ell(n)}{2 \tilde{\ell}(n)} \quad(n \rightarrow \infty) ;
$$

(3) if $-\infty<d \leq 0$, and if futher $\int^{\infty} \ell(s) d s / s<\infty$ for $d=0$, then

$$
|\alpha(n)| \sim \frac{n^{2 d-1} \ell(n)}{\sum_{-\infty}^{\infty} \gamma(k)} \quad(n \rightarrow \infty) .
$$

We must point out that what we actually do below is to prove (2.7)-(2.9) separately rather than to prove (2.6) directly. It is easy to show that the asymptotics (2.7)-(2.9) are unified in (2.6) but it is still mysterious why this is so.

\section{INTERSECTION OF PAST AND FUTURE}

In this section, we prove a discrete analogue of the Seghier-Dym theorem ([S], [Dy2]; see also Levinson-McKean [LM, §6c], Dym-McKean [DM, §4.3] and Dym [Dy1, Theorem 2.1]). It plays a crucial role in this paper though it is used only once, viz. in the proof of Theorem 4.1 below. Note that, as stated in $\S 2$, the stationary process $\left\{X_{n}\right\}$ is assumed to be purely nondeterministic.

Theorem 3.1. If the spectral density $\Delta(\cdot)$ of $\left\{X_{n}\right\}$ satisfies $\int_{-\pi}^{\pi} \Delta(\theta)^{-1} d \theta<\infty$, then

$$
H_{(-\infty, 0]} \cap H_{[-n, \infty)}=H_{[-n, 0]}
$$

holds for every $n \geq 0$.

Proof. Step 1. We denote by $H^{\mathbb{C}}$ the closed complex linear hull of $\left\{X_{k}: k \in\right.$ $\mathbb{Z}\}$ in $L^{2}(\Omega, \mathcal{F}, P)$. Then $H^{\mathbb{C}}$ is a complex Hilbert space with inner product $\left(Y_{1}, Y_{2}\right):=E\left[Y_{1} \overline{Y_{2}}\right]$. We define its closed subspaces $H_{(-\infty, 0]}^{\mathbb{C}}, H_{[-n, \infty)}^{\mathbb{C}}$ and $H_{[-n, 0]}^{\mathbb{C}}$ as we defined $H_{(-\infty, 0]}, H_{[-n, \infty)}$ and $H_{[-n, 0]}$ in $\S 2$, but replacing $\mathbb{R}$ by $\mathbb{C}$. We prove

$$
H_{(-\infty, 0]}^{\mathbb{C}} \cap H_{[-n, \infty)}^{\mathbb{C}}=H_{[-n, 0]}^{\mathbb{C}} \quad \text { for all } n \geq 0 .
$$

The assertion (3.1) for the real case follows from this. 
We write $L$ for the complex Hilbert space $L^{2}((-\pi, \pi), \Delta(\theta) d \theta)$ with the inner product

$$
(f, g)_{L}:=\int_{-\pi}^{\pi} f(\theta) \overline{g(\theta)} \Delta(\theta) d \theta
$$

For $I \subset \mathbb{Z}$, we denote by $L_{I}$ the closed complex linear hull of $\left\{e^{i k \theta}: k \in I\right\}$ in $L$. In particular, for $m \in \mathbb{Z}$ and $n \in \mathbb{Z}$ with $m \leq n$, we write $L_{(-\infty, m]}, L_{[m, \infty)}$ and $L_{[m, n]}$ for $L_{I}$ with $I=\{k \in \mathbb{Z}:-\infty<k \leq m\},\{k \in \mathbb{Z}: m \leq k<\infty\}$ and $\{k \in \mathbb{Z}: m \leq k \leq n\}$, respectively.

The stationary process $\left\{X_{n}\right\}$ permits a spectral representation of the form

$$
X(k)=\int_{-\pi}^{\pi} e^{i k \theta} Z(d \theta) \quad(n \in \mathbb{Z}),
$$

where $Z$ is the spectral measure such that $E[Z(A) \overline{Z(B)}]=\int_{A \cap B} \Delta(\theta) d \theta$ (see $[\mathrm{BD}, \S 4.8])$. The mapping $f \mapsto \int_{-\pi}^{\pi} f(\theta) Z(d \theta)$ gives a Hilbert space isomorphism of $L$ onto $H^{\mathbb{C}}$. For $I \subset \mathbb{Z}$, the subspace $L_{I}$ is mapped to $H_{I}^{\mathbb{C}}$. So in order to prove (3.2), it is enough to prove

$$
L_{(-\infty, 0]} \cap L_{[-n, \infty)}=L_{[-n, 0]} \text { for all } n \geq 0 .
$$

However, the implication $\supset$ is trivial; hence we prove only the opposite one $(\subset)$.

We write $H^{2+}$ for the Hardy space $H^{2+}$ of class 2 over the unit disk $|z|<1$, and $H^{2-}$ for that over the region $|z|>1$ of the Riemann sphere $\mathbb{C} \cup\{\infty\}$. As usual, we identify each function $f(z)$ in $H^{2+}$ or $H^{2-}$ with its boundary function $f\left(e^{i \theta}\right)$ and regard both $H^{2+}$ and $H^{2-}$ as subspaces of $L^{2}((-\pi, \pi), d \theta)$.

We define an outer function $h$ in $H^{2+}$ by $(2.1)$, and $h^{*} \in H^{2-}$ by $h^{*}(z):=$ $\overline{h(1 / \bar{z})}(|z|>1)$. We define $h_{n} \in H^{2+}$ by $h_{n}(z):=z^{n} h(z)$. Then since $L_{[-n, \infty)}=$ $e^{-i n \theta} L_{[0, \infty)}$, it follows that

$$
L_{[-n, \infty)}=\frac{1}{h_{n}} H^{2+}, \quad L_{(-\infty, 0]}=\frac{1}{h^{*}} H^{2-}
$$

(see Ibragimov and Rozanov [IR, II.2, Theorem 1]; Beurling's theorem is essential here). So, for any $f\left(e^{i \theta}\right) \in L_{(-\infty, 0]} \cap L_{[-n, \infty)}$, there exist $g_{+} \in H^{2+}$ and $g_{-} \in H^{2-}$ such that

$$
f\left(e^{i \theta}\right)=\frac{g_{+}\left(e^{i \theta}\right)}{h_{n}\left(e^{i \theta}\right)}=\frac{g_{-}\left(e^{i \theta}\right)}{h^{*}\left(e^{i \theta}\right)} \quad \text { a.e. on }(-\pi, \pi) .
$$

For these $g_{+}$and $g_{-}$, we put $f(z):=g_{+}(z) / h_{n}(z)$ for $|z|<1$, and $f(z):=$ $g_{-}(z) / h^{*}(z)$ for $|z|>1$. Then $f$ is meromorphic in $|z|<1$ and possibly has a 
unique pole at zero, of order at most $n$, while $f$ is holomorphic in the region $|z|>1$ of the Riemann sphere. We claim that the function $f$ can be continued analytically from $|z|<1$ to $|z|>1$ across the unit circle $|z|=1$.

This claim implies that the function $f$ so obtained is meromorphic over the Riemann sphere, whence it is a rational function. By the above description of singularity, $f$ must be of the form $f(z)=\sum_{k=0}^{n} a_{k} z^{-k}$ with some $a_{k} \in \mathbb{C}$ $(k=1, \ldots, n)$, whence $f\left(e^{i \theta}\right)=\sum_{k=0}^{n} a_{k} e^{-i k \theta}$. Therefore $f\left(e^{i \theta}\right) \in L_{[-n, 0]}$, and this gives the desired implication $L_{(-\infty, 0]} \cap L_{[-n, \infty)} \subset L_{[-n, 0]}$.

Step 2. We complete the proof by proving the claim above. It should be pointed out that the argument below is parallel to that of [LM, $\S 6 \mathrm{c}]$.

Put $A_{k}:=\left\{\theta \in(-\pi, \pi): \sup _{1-(1 / k)<r<1}\left|f\left(r e^{i \theta}\right)\right| \geq k\right\}$ for $k=1,2, \ldots$ Then $A_{k} \supset A_{k+1}(k=1,2, \ldots)$. Moreover, since

$$
\int_{-\pi}^{\pi}\left|f\left(e^{i \theta}\right)\right| d \theta \leq\left\{\int_{-\pi}^{\pi}\left|f\left(e^{i \theta}\right)\right|^{2} \Delta(\theta) d \theta\right\}^{1 / 2}\left\{\int_{-\pi}^{\pi} \Delta(\theta)^{-1} d \theta\right\}^{1 / 2}<\infty,
$$

Egoroff's theorem implies that the Lebesgue measure of $A_{k}$ tends to zero as $k \rightarrow \infty$.

Now we have

$$
\begin{aligned}
\int_{A_{k}}\left|f\left(r e^{i \theta}\right)\right| d \theta & \leq r^{-n}\left\{\int_{A_{k}}\left|g_{+}\left(r e^{i \theta}\right)\right|^{2} d \theta\right\}^{1 / 2}\left\{\int_{A_{k}}\left|h\left(r e^{i \theta}\right)\right|^{-2} d \theta\right\}^{1 / 2} \\
& \leq r^{-n}\left\|g_{+}\right\|_{2+}\left\{\int_{A_{k}}\left|h\left(r e^{i \theta}\right)\right|^{-2} d \theta\right\}^{1 / 2}
\end{aligned}
$$

where $\left\|g_{+}\right\|_{2+}$ is the $H^{2+}$-norm of $g_{+}$. Let

$$
P_{r}(\theta):=\frac{1-r^{2}}{1-2 r \cos \theta+r^{2}}
$$

be the Poisson kernel. By Jensen's inequality,

$$
\begin{aligned}
\left|h\left(r e^{i \theta}\right)\right|^{-2} & =\frac{1}{2 \pi} \exp \left\{\frac{1}{2 \pi} \int_{-\pi}^{\pi} P_{r}(\theta-t) \log \Delta(t)^{-1} d t\right\} \\
& \leq \frac{1}{(2 \pi)^{2}} \int_{-\pi}^{\pi} P_{r}(\theta-t) \Delta(t)^{-1} d t
\end{aligned}
$$

whence, for $k \geq 2$,

$$
\begin{aligned}
& \sup _{1-(1 / k)<r<1} \int_{A_{k}}\left|f\left(r e^{i \theta}\right)\right| d \theta \\
& \leq \frac{2^{n}\left\|g_{+}\right\|_{2+}}{(2 \pi)^{1 / 2}}\left[\int_{-\pi}^{\pi}\left\{\sup _{1-(1 / k)<r<1} \frac{1}{2 \pi} \int_{A_{k}} P_{r}(t-\theta) d \theta\right\} \Delta(t)^{-1} d t\right]^{1 / 2} .
\end{aligned}
$$


For $m \in \mathbb{N}$ and for almost all $t \in(-\pi, \pi)$, we have

$$
\begin{aligned}
0 & \leq \limsup _{k \rightarrow \infty} \sup _{1-(1 / k)<r<1} \frac{1}{2 \pi} \int_{A_{k}} P_{r}(t-\theta) d \theta \\
& \leq \lim _{k \rightarrow \infty} \sup _{1-(1 / k)<r<1} \frac{1}{2 \pi} \int_{A_{m}} P_{r}(t-\theta) d \theta=I_{A_{m}}(t) .
\end{aligned}
$$

Let $m \rightarrow \infty$. Then we obtain

$$
\lim _{k \rightarrow \infty} \sup _{1-(1 / k)<r<1} \frac{1}{2 \pi} \int_{A_{k}} P_{r}(t-\theta) d \theta=0 \quad \text { a.e. on }(-\pi, \pi) .
$$

Consequently,

$$
\lim _{k \rightarrow \infty} \sup _{1-(1 / k)<r<1} \int_{A_{k}}\left|f\left(r e^{i \theta}\right)\right| d \theta=0
$$

so that

$$
\lim _{r \uparrow 1} \int_{-\pi}^{\pi}\left|f\left(e^{i \theta}\right)-f\left(r e^{i \theta}\right)\right| d \theta=0 .
$$

The analogous result for $r \downarrow 1$ follows similarly from the fact $g_{-} \in H^{2-}$.

Choose $\alpha \in(-\pi, \pi)$ so that $f\left(r e^{i \alpha}\right)$ tends boundedly to $f\left(e^{i \alpha}\right)$ as $r \rightarrow 1$. For $z=r e^{i \theta}$ in the region $D:=\left\{r e^{i \theta}: \frac{1}{2}<r<2, \alpha<\theta<\alpha+2 \pi\right\}$, define $F(z)$ by $F(z):=\int_{\Gamma} f(w) d w$, where the path $\Gamma=\gamma_{1}+\gamma_{2}$ from $e^{i \alpha}$ to $z$ is defined by $\gamma_{1}(t):=t e^{i \alpha}$ with $t$ from 1 to $r$ and then $\gamma_{2}(t):=r e^{i t}$ with $t$ from $\alpha$ to $\theta$. Then the function $F$ is holomorphic in $D_{0}:=D \backslash\{z \in D:|z|=1\}$ and continuous in $D$, whence $F$ is holomorphic in $D$ by the reflection principle. Since $f=F^{\prime}$ in $D_{0}$, this implies that $f$ can be continued analytically in $D$ across $|z|=1$. Since we can choose a different $\alpha$ and do the same thing, we conclude that $f$ can be continued analytically across the whole unit circle $|z|=1$, as claimed. This completes the proof.

\section{REPRESENTATIONS}

In this section, we establish some representation theorems in terms of the $\mathrm{MA}(\infty)$ coefficients $c_{n}$ and $\operatorname{AR}(\infty)$ coefficients $a_{n}$ for a purely nondeterministic stationary process $\left\{X_{n}\right\}$. These enable us to carry out the asymptotic analysis via $\left\{c_{n}\right\}$ and $\left\{a_{n}\right\}$ in $\S 6$.

For $Y \in H$ and $I \subset \mathbb{Z}$, we may think of $P_{I} Y$ as the best predictor of $Y$ on the observations $\left\{X_{k}: k \in I\right\}$, whence $P_{I}^{\perp} Y=Y-P_{I} Y$ as its prediction error. 
From the orthogonal decompositions

$$
P_{[-n, 0]}^{\perp}=P_{(-\infty, 0]}^{\perp}+P_{[-n, 0]}^{\perp} P_{(-\infty, 0]}=P_{[-n, \infty)}^{\perp}+P_{[-n, 0]}^{\perp} P_{[-n, \infty)},
$$

we have

$$
\begin{aligned}
\left\|P_{[-n, 0]}^{\perp} Y\right\|^{2}= & \left\|P_{(-\infty, 0]}^{\perp} Y\right\|^{2}+ \\
= & \left\|P_{(-\infty, 0]}^{\perp} Y\right\|^{2}+ \\
& +\left\|P_{[-n, 0]}^{\perp} P_{(-\infty, 0]}^{\perp} Y\right\|_{(-\infty, 0]} Y \|^{2} \\
& +\left\|P_{[-n, 0]}^{\perp} P_{[-n, \infty)} P_{(-\infty, 0]} Y\right\|^{2}
\end{aligned}
$$

and similarly, by induction, for $m \geq 0$,

$$
\begin{aligned}
\left\|P_{[-n, 0]}^{\perp} Y\right\|^{2}=\sum_{k=0}^{m} & \left\|P_{(-\infty, 0]}^{\perp}\left\{P_{[-n, \infty)} P_{(-\infty, 0]}\right\}^{k} Y\right\|^{2} \\
& +\sum_{k=0}^{m}\left\|P_{[-n, \infty)}^{\perp}\left\{P_{(-\infty, 0]} P_{[-n, \infty)}\right\}^{k} P_{(-\infty, 0]} Y\right\|^{2} \\
& +\left\|P_{[-n, 0]}^{\perp}\left\{P_{[-n, \infty)} P_{(-\infty, 0]}\right\}^{m+1} Y\right\|^{2} .
\end{aligned}
$$

Now what will happen if we let $m \rightarrow \infty$ ? The following theorem gives an answer.

Theorem 4.1. If the spectral density $\Delta(\cdot)$ of $X$ satisfies $\int_{-\pi}^{\pi} \Delta(\theta)^{-1} d \theta<\infty$, then for $Y \in H$ and $n \geq 0$,

$$
\begin{aligned}
\left\|P_{[-n, 0]}^{\perp} Y\right\|^{2}= & \sum_{k=0}^{\infty}\left\|P_{(-\infty, 0]}^{\perp}\left\{P_{[-n, \infty)} P_{(-\infty, 0]}\right\}^{k} Y\right\|^{2} \\
& +\sum_{k=0}^{\infty}\left\|P_{[-n, \infty)}^{\perp}\left\{P_{(-\infty, 0]} P_{[-n, \infty)}\right\}^{k} P_{(-\infty, 0]} Y\right\|^{2} .
\end{aligned}
$$

Proof. It follows from Theorem 3.1 that $H_{(-\infty, 0]} \cap H_{[-n, \infty)}=H_{[-n, 0]}$. Hence,

$$
\operatorname{s-lim}_{m \rightarrow \infty}\left\{P_{(-\infty, 0]} P_{[-n, \infty)}\right\}^{m}=P_{[-n, 0]}
$$

(see, for example, Halmos [Ha, Problem 122]). This implies that the last term of the right-hand side of (4.2) tends to zero as $m \rightarrow \infty$. Thus the theorem follows.

The point of Theorem 4.1 is that it enables us to investigate the prediction problem from a finite part of time via the prediction from an infinite past and that from an infinite future.

We can give the key assumption $\int_{-\pi}^{\pi} \Delta(\theta)^{-1} d \theta<\infty$ above in different ways.

Proposition 4.2. The following conditions are equivalent:

(1) $\int_{-\pi}^{\pi} \Delta(\theta)^{-1} d \theta<\infty$; 
(2) $h^{-1} \in H^{2+}$;

(3) $\sum_{0}^{\infty}\left(a_{n}\right)^{2}<\infty$.

Proof. The implication $(2) \Leftrightarrow(3)$ follows from the well-known characterization of the space $H^{2+}$ in terms of power series coefficients (see [Ru, Theorem 17.12]). On the other hand, since

$$
\frac{1}{h(z)}=(2 \pi)^{-1 / 2} \exp \left\{\frac{1}{4 \pi} \int_{-\pi}^{\pi} \frac{e^{i \theta}+z}{e^{i \theta}-z} \log \{1 / \Delta(\theta)\} d \theta\right\} \quad(|z|<1),
$$

we see that (1) and (2) are equivalent (see [Ru, Theorem 17.16]).

We look at the relation between the condition above and those in $\S 2$.

Proposition 4.3. If $(\mathrm{C} 1)$ and (A1) hold, then we have $\sum_{0}^{\infty}\left|a_{n}\right|<\infty$ and hence $\int_{-\pi}^{\pi} \Delta(\theta)^{-1} d \theta<\infty$.

Proof. Bearing in mind that $\left\{a_{n}\right\}$ is eventually non-negative, we apply the monotone convergence theorem to (2.2). Then it follows that

$$
\sum_{n=0}^{\infty} a_{n}=-\lim _{r \uparrow 1}\left(\sum_{n=0}^{\infty} c_{n} r^{n}\right)^{-1} \in(-\infty, 0]
$$

which implies $\sum_{0}^{\infty}\left|a_{n}\right|<\infty$. In particular, we have $\sum_{0}^{\infty}\left(a_{n}\right)^{2}<\infty$, so that, by Proposition $4.2, \int_{-\pi}^{\pi} \Delta(\theta)^{-1} d \theta<\infty$.

Now we consider $P_{(-\infty, 0]} X_{n}$ for $n \geq 1$. We vaguely think of it as a linear combination of $\left\{X_{k}:-\infty<k \leq 0\right\}$. We make this point clear to the extent sufficient for our purpose. We put

$$
b_{j}^{m}:=\sum_{k=1}^{m} c_{m-k} a_{k+j} \quad(m \geq 1, \quad j \geq 0) .
$$

Theorem 4.4. If $\sum_{0}^{\infty}\left|a_{k}\right|<\infty$, then for $n \in \mathbb{N}$,

$$
P_{(-\infty, 0]} X_{n}=\sum_{j=0}^{\infty} b_{j}^{n} X_{-j}
$$

the sum converging absolutely in $H$.

Proof. Recall the spectral representation (3.3) for the stationary process $\left\{X_{n}\right\}$. Since $\left|h\left(e^{i \theta}\right)\right|^{2}=2 \pi \Delta(\theta)>0$ a.e. on $(-\pi, \pi)$, we may put

$$
\xi_{n}:=\int_{-\pi}^{\pi} e^{i n \theta}\left\{\overline{h\left(e^{i \theta}\right)}\right\}^{-1} Z(d \theta) \quad(n \in \mathbb{Z}) .
$$


Then, as is well known, $\left\{\xi_{n}: n \in \mathbb{Z}\right\}$ forms a complete orthonormal system for $H$ such that

$$
X_{n}=\sum_{j=-\infty}^{n} c_{n-j} \xi_{j}, \quad H_{(-\infty, n]}=H_{(-\infty, n]}(\xi) \quad(n \in \mathbb{Z}),
$$

where $H_{(-\infty, n]}(\xi)$ is the closed subspace of $H$ spanned by $\left\{\xi_{k}:-\infty<k \leq\right.$ $n\}$ (see [Ro, Chapter II]). The representation (4.7) is the so-called canonical representation of $\left\{X_{n}\right\}$. It follows that

$$
\left\|\sum_{k=0}^{m} a_{k} X_{n-k}+\xi_{n}\right\|^{2}=\int_{-\pi}^{\pi}\left|f_{m}(\theta)\right|^{2} \Delta(\theta) d \theta \quad(m \in \mathbb{N}),
$$

where

$$
f_{m}(\theta):=\frac{1}{h\left(e^{i \theta}\right)}+\sum_{k=0}^{m} a_{k} e^{i k \theta} \quad(-\pi<\theta<\pi) .
$$

By assumption, $h^{-1}(\cdot)$ is in $H^{2+}$. Hence we have the Fourier series expansion

$$
\frac{1}{h\left(e^{i \theta}\right)}=-\sum_{k=0}^{\infty} a_{k} e^{i k \theta}
$$

in $L^{2}((-\pi, \pi), d \theta)$, which yields $f_{m}(\theta)=-\sum_{m+1}^{\infty} a_{k} e^{i k \theta}$. The condition $\sum_{0}^{\infty}\left|a_{k}\right|<$ $\infty$ now implies that $f_{m}(\theta)$ tends boundedly to zero as $m \rightarrow \infty$, and so the righthand side of (4.8) converges to zero as $m \rightarrow \infty$. Thus we obtain the following $\operatorname{AR}(\infty)$ representation for $\left\{X_{n}\right\}$ :

$$
\sum_{j=-\infty}^{n} a_{n-j} X_{j}+\xi_{n}=0 \quad(n \in \mathbb{Z}) .
$$

We set $Y_{n}:=P_{(-\infty, 0]} X_{n}$ for $n \in \mathbb{N}$. By (4.9), the sequence $\left\{Y_{n}: n \in \mathbb{N}\right\}$ is a solution to

$$
\sum_{m=1}^{n} a_{n-m} Y_{m}=-\sum_{j=0}^{\infty} a_{n+j} X_{-j} \quad(n \in \mathbb{N}) .
$$

On the other hand, by (2.3),

$$
\begin{array}{r}
\sum_{m=1}^{n} a_{n-m} \sum_{j=0}^{\infty} b_{j}^{m} X_{-j}=\sum_{j=0}^{\infty}\left(\sum_{m=1}^{n} a_{n-m} \sum_{k=1}^{m} c_{m-k} a_{k+j}\right) X_{-j} \\
=\sum_{j=0}^{\infty}\left(\sum_{k=1}^{n} a_{k+j} \sum_{p=0}^{n-k} a_{n-k-p} c_{p}\right) X_{-j}=-\sum_{j=0}^{\infty} a_{n+j} X_{-j}
\end{array}
$$

which implies that the sequence $\left\{\sum_{j=0}^{\infty} b_{j}^{n} X_{-j}: n \in \mathbb{N}\right\}$ is also a solution to (4.10). However, the solution to (4.10) is unique because $a_{0} \neq 0$. Thus (4.5) follows. 
Since the stationary process $\left\{X_{n}\right\}$ is assumed to be purely nondeterministic, we have $P_{(-\infty, 0]}^{\perp} X_{1} \neq 0$; use (4.16) below and the fact $c_{0} \neq 0$. So we put

$$
\epsilon(n):=\frac{\left\|P_{[-n, 0]}^{\perp} X_{1}\right\|^{2}-\left\|P_{(-\infty, 0]}^{\perp} X_{1}\right\|^{2}}{\left\|P_{(-\infty, 0]}^{\perp} X_{1}\right\|^{2}} \quad(n=0,1, \ldots) .
$$

We note that $\epsilon(n) \rightarrow 0$ as $n \rightarrow \infty$ because, by (4.1) and (4.7),

$$
\begin{aligned}
\left\|P_{[-n, 0]}^{\perp} X_{1}\right\|^{2} & -\left\|P_{(-\infty, 0]}^{\perp} X_{1}\right\|^{2}=\left\|P_{[-n, 0]}^{\perp} \sum_{j=n+2}^{\infty} c_{j} \xi_{1-j}\right\|^{2} \\
& \leq\left\|\sum_{j=n+2}^{\infty} c_{j} \xi_{1-j}\right\|^{2}=\sum_{j=n+2}^{\infty}\left(c_{j}\right)^{2} \rightarrow 0 \quad(n \rightarrow \infty) .
\end{aligned}
$$

In $\S 6$, we give a detailed treatment of the asymptotic behaviour of $\epsilon(n)$ as $n \rightarrow \infty$. Here we prove a representation of $\epsilon(n)$ in terms $\left\{c_{k}\right\}$ and $\left\{a_{k}\right\}$.

Theorem 4.5. If $\sum_{0}^{\infty}\left|a_{k}\right|<\infty$, then for $n \in \mathbb{N}$,

$$
\epsilon(n)=\sum_{k=1}^{\infty} \sum_{p=0}^{\infty} d_{k}(n, p)^{2}
$$

where $d_{1}(n, p):=\sum_{v=0}^{\infty} c_{v} a_{v+n+2+p}$ and for $k \geq 2$,

$$
d_{k}(n, p):=\sum_{m_{1}=1}^{\infty} a_{n+1+m_{1}} \sum_{m_{2}=1}^{\infty} b_{n+m_{2}}^{m_{1}} \ldots \sum_{m_{k-1}=1}^{\infty} b_{n+m_{k-1}}^{m_{k-2}} \sum_{m_{k}=1}^{\infty} b_{n+p+m_{k}}^{m_{k-1}} c_{m_{k}-1} .
$$

Proof. By Theorem 4.4, we have

$$
P_{(-\infty, 0]} X_{m}=\sum_{j=1}^{\infty} b_{n+j}^{m} X_{-n-j} \quad\left(\bmod H_{[-n, 0]}\right)
$$

for $m \geq 1$ and $n \geq 0$. Let $S_{k}$ and $\theta$ be the $k$-step shift operator and reflection operator on $H$ :

$$
S_{k}\left(X_{m}\right)=X_{m+k}, \quad \theta\left(X_{m}\right)=X_{-m}
$$

Then $S_{k}$ and $\theta$ are Hilbert space automorphisms of $H$ such that $S_{k}^{-1}=S_{-k}$, $\theta^{-1}=\theta$. In view of the identity $\left(\theta S_{n}\right)^{-1} P_{(-\infty, 0]}\left(\theta S_{n}\right)=P_{[-n, \infty)}$, we have

$$
P_{[-n, \infty)} X_{-n-m}=\sum_{j=1}^{\infty} b_{n+j}^{m} X_{j} \quad\left(\bmod H_{[-n, 0]}\right) .
$$

Hence

$$
\begin{gathered}
\left\{P_{[-n, \infty)} P_{(-\infty, 0]}\right\}^{k} X_{1}=c_{0} \sum_{m_{1}=1}^{\infty} a_{n+1+m_{1}} \sum_{m_{2}=1}^{\infty} b_{n+m_{2}}^{m_{1}} \\
\cdots \sum_{m_{r-1}=1}^{\infty} b_{n+m_{r-1}}^{m_{r-2}} \sum_{m_{r}=1}^{\infty} b_{n+m_{r}}^{m_{r-1}} X_{m_{r}} \quad\left(\bmod H_{[-n, 0]}\right),
\end{gathered}
$$


where $r:=2 k$. This and

$$
P_{(-\infty, 0]}^{\perp} X_{m}=\sum_{q=1}^{m} c_{m-q} \xi_{q} \quad(m \in \mathbb{N})
$$

yield

$$
\begin{aligned}
& P_{(-\infty, 0]}^{\perp}\left\{P_{[-n, \infty)} P_{(-\infty, 0]}\right\}^{k} X_{1} \\
= & c_{0} \sum_{m_{1}=1}^{\infty} a_{n+1+m_{1}} \sum_{m_{2}=1}^{\infty} b_{n+m_{2}}^{m_{1}} \cdots \sum_{m_{r-1}=1}^{\infty} b_{n+m_{r-1}}^{m_{r-2}} \sum_{m_{r}=1}^{\infty} b_{n+m_{r}}^{m_{r-1}} \sum_{q=1}^{m_{r}} c_{m_{r}-q} \xi_{q} .
\end{aligned}
$$

Therefore, for $p \geq 0$,

$$
\left(P_{(-\infty, 0]}^{\perp}\left\{P_{[-n, \infty)} P_{(-\infty, 0]}\right\}^{k} X_{1}, \xi_{p+1}\right)=c_{0} d_{2 k}(n, p)
$$

so that

$$
\begin{aligned}
& \left\|P_{(-\infty, 0]}^{\perp}\left\{P_{[-n, \infty)} P_{(-\infty, 0]}\right\}^{k} X_{1}\right\|^{2} \\
= & \sum_{p=0}^{\infty}\left(P_{(-\infty, 0]}^{\perp}\left\{P_{[-n, \infty)} P_{(-\infty, 0]}\right\}^{k} X_{1}, \xi_{p+1}\right)^{2}=\left(c_{0}\right)^{2} \sum_{p=0}^{\infty} d_{2 k}(n, p)^{2} .
\end{aligned}
$$

Similarly, we obtain

$$
\begin{aligned}
& \left\|P_{[-n, \infty)}^{\perp}\left\{P_{(-\infty, 0]} P_{[-n, \infty)}\right\}^{k} P_{(-\infty, 0]} X_{1}\right\|^{2} \\
= & \left\|P_{[-n, \infty)}^{\perp} P_{(-\infty, 0]}\left\{P_{[-n, \infty)} P_{(-\infty, 0]}\right\}^{k} X_{1}\right\|^{2}=\left(c_{0}\right)^{2} \sum_{p=0}^{\infty} d_{2 k+1}(n, p)^{2} .
\end{aligned}
$$

Since we have $\int_{-\pi}^{\pi} \Delta(\theta)^{-1} d \theta<\infty$ by Proposition 4.2 , it follows from Theorem 4.1 that

$$
\left\|P_{[-n, 0]}^{\perp} X_{1}\right\|^{2}=\left\|P_{(-\infty, 0]}^{\perp} X_{1}\right\|^{2}+\left(c_{0}\right)^{2} \sum_{k=1}^{\infty} \sum_{p=0}^{\infty} d_{k}(n, p)^{2} .
$$

However,

$$
\left\|P_{(-\infty, 0]}^{\perp} X_{1}\right\|^{2}=\left\|c_{0} \xi_{1}\right\|^{2}=\left(c_{0}\right)^{2}
$$

whence (4.12) follows.

Theorem 4.6. We assume $(\mathrm{C} 1)$ and $(\mathrm{A} 1)$, and choose $M \in \mathbb{N}$ so that $a_{n+2} \geq 0$ for all $n \geq M$. Then, for $d_{k}(n, p)$ in Theorem 4.5 with $n \geq M$ and $p \geq 0$, we have

$$
d_{2}(n, p)=\sum_{v_{2}=0}^{\infty} c_{v_{2}} \sum_{v_{1}=0}^{\infty} c_{v_{1}} \sum_{m=0}^{\infty} a_{v_{2}+m+n+2+p} a_{v_{1}+m+n+2}
$$


and for $k \geq 3$,

$$
\begin{aligned}
d_{k}(n, p) & =\sum_{v_{k}=0}^{\infty} c_{v_{k}} \sum_{v_{k-1}=0}^{\infty} c_{v_{k-1}} \\
& \ldots \sum_{v_{1}=0}^{\infty} c_{v_{1}} \sum_{m_{k-1}=0}^{\infty} a_{v_{k}+m_{k-1}+n+2+p} \sum_{m_{k-2}=0}^{\infty} a_{v_{k-1}+m_{k-1}+m_{k-2}+n+2} \\
& \ldots \sum_{m_{2}=0}^{\infty} a_{v_{3}+m_{3}+m_{2}+n+2} \sum_{m_{1}=0}^{\infty} a_{v_{2}+m_{2}+m_{1}+n+2} a_{v_{1}+m_{1}+n+2} .
\end{aligned}
$$

Proof. We first note that, by Proposition 4.3, $\sum_{0}^{\infty}\left|a_{k}\right|<\infty$ holds. By assumption, we can apply the Fubini-Tonelli theorem to exchange the order of sums. In particular, for $n \geq M$,

$$
\begin{gathered}
d_{k}(n, p)=\sum_{v_{k}=0}^{\infty} c_{v_{k}} \sum_{m_{k-1}=0}^{\infty} b_{n+1+v_{k}+p}^{m_{k-1}+1} \sum_{m_{k-2}=0}^{\infty} b_{n+1+m_{k-1}}^{m_{k-2}+1} \\
\cdots \sum_{m_{2}=0}^{\infty} b_{n+1+m_{3}}^{m_{2}+1} \sum_{m_{1}=0}^{\infty} b_{n+1+m_{2}}^{m_{1}+1} a_{m_{1}+n+2} .
\end{gathered}
$$

Since $b_{j+1}^{m+1}=\sum_{v=0}^{m} c_{v} a_{m-v+j+2}$ for $m \geq 0$ and $j \geq 0$, it follows that

$$
\begin{aligned}
\sum_{m_{1}=0}^{\infty} b_{n+1+m_{2}}^{m_{1}+1} a_{m_{1}+n+2} & =\sum_{m_{1}=0}^{\infty}\left(\sum_{v_{1}=0}^{m_{1}} c_{v_{1}} a_{m_{1}-v_{1}+m_{2}+n+2}\right) a_{m_{1}+n+2} \\
& =\sum_{v_{1}=0}^{\infty} c_{v_{1}} \sum_{m_{1}=0}^{\infty} a_{m_{2}+m_{1}+n+2} a_{v_{1}+m_{1}+n+2} .
\end{aligned}
$$

This gives (4.17). Similarly

$$
\begin{aligned}
\sum_{m_{2}=0}^{\infty} & b_{n+1+m_{3}}^{m_{2}+1} \sum_{m_{1}=0}^{\infty} b_{n+1+m_{2}}^{m_{1}+1} a_{m_{1}+n+2} \\
& =\sum_{v_{1}=0}^{\infty} c_{v_{1}} \sum_{m_{2}=0}^{\infty}\left(\sum_{v_{2}=0}^{m_{2}} c_{v_{2}} a_{m_{2}-v_{2}+m_{3}+n+2}\right) \sum_{m_{1}=0}^{\infty} a_{m_{2}+m_{1}+n+2} a_{v_{1}+m_{1}+n+2} \\
& =\sum_{v_{2}=0}^{\infty} c_{v_{2}} \sum_{v_{1}=0}^{\infty} c_{v_{1}} \sum_{m_{2}=0}^{\infty} a_{m_{3}+m_{2}+n+2} \sum_{m_{1}=0}^{\infty} a_{v_{2}+m_{2}+m_{1}+n+2} a_{v_{1}+m_{1}+n+2} .
\end{aligned}
$$

Repeating the same arguments, we arrive at (4.18).

\section{Asymptotic Relations}

The aim of this section is to give the link among the asymptotics for the autocovariance function $\gamma(\cdot)$, spectral density $\Delta(\cdot)$, sequence $\left\{c_{n}\right\}$ of $\mathrm{MA}(\infty)$ coefficients, and sequence $\left\{a_{n}\right\}$ of $\operatorname{AR}(\infty)$ coefficients for a purely nondeterministic stationary process $\left\{X_{n}\right\}$. We refer to [I1]-[I6] for related work. 
Since the spectral density $\Delta(\cdot)$ of $\left\{X_{n}\right\}$ is an even function, we have

$$
\begin{gathered}
\gamma(n)=2 \int_{0}^{\pi} \Delta(\theta) \cos (n \theta) d \theta \quad(n \in \mathbb{Z}), \\
\sum_{n=0}^{\infty} c_{n} r^{n}=(2 \pi)^{1 / 2} \exp \left\{\frac{1}{4 \pi} \int_{-\pi}^{\pi} P_{r}(\theta) \log \Delta(\theta) d \theta\right\} \quad(-1<r<1)
\end{gathered}
$$

(recall $P_{r}(\theta)$ from $(3.4)$ ). On the other hand, it follows from (4.7) that

$$
\gamma(n)=\sum_{m=0}^{\infty} c_{n+m} c_{m} \quad(n \geq 0)
$$

If the sequence $\left\{c_{n}\right\}$ satisfies $(\mathrm{C} 1)$ and $(\mathrm{C} 2)$, then the sequence $\{\gamma(n)\}_{0}^{\infty}$ is eventually decreasing to zero; and so the Fourier series

$$
\frac{1}{2 \pi} \gamma(0)+\frac{1}{\pi} \sum_{n=1}^{\infty} \gamma(n) \cos (n \theta)
$$

converges to a continuous function on $(-\pi, \pi) \backslash\{0\}$ (see Zygmund [Z, Chapter I, (2.6)]). Moreover, by Lebesgue's theorem ([Z, Chapter III, (3.9)]), the Fourier series coincides with $\Delta(\theta)$ almost everywhere. So in the sequel, we identify $\Delta(\theta)$ with the Fourier series (5.4).

To state the result for the boundary case, we recall the notion of $\Pi$-variation. For $\ell \in \mathcal{R}_{0}$, the class $\Pi_{\ell}$ is the class of real-valued measurable $g$, defined on some neighbourhood $[A, \infty)$ of infinity, such that

$$
\lim _{x \rightarrow \infty}\{g(\lambda x)-g(x)\} / \ell(x)=c \log \lambda \quad \text { for all } \lambda>0
$$

with $c \in \mathbb{R}$ called the $\ell$-index of $g$ (see [BGT, Chapter 3] for background).

The following theorems are the results for the long-memory processes, boundary case, and intermediate-memory processes ([BD, p. 520]), respectively.

Theorem 5.1. Let $\ell \in \mathcal{R}_{0}$ and $0<d<\frac{1}{2}$. We assume $(\mathrm{C} 1)$ and $(\mathrm{C} 2)$. Then (2.5) and the following are equivalent:

$$
\begin{aligned}
& \Delta(\theta) \sim \theta^{-2 d} \ell(1 / \theta) \cdot \frac{1}{2 \Gamma(1-2 d) \sin (\pi d)} \quad(\theta \rightarrow 0+), \\
& c_{n} \sim n^{-(1-d)}\left\{\frac{\ell(n)}{B(d, 1-2 d)}\right\}^{1 / 2} \quad(n \rightarrow \infty) .
\end{aligned}
$$

If we further assume (A1), then each of these conditions implies that

$$
a_{n} \sim n^{-(1+d)}\left\{\frac{\ell(n)}{B(d, 1-2 d)}\right\}^{-1 / 2} \frac{d \sin (\pi d)}{\pi} \quad(n \rightarrow \infty) .
$$


Theorem 5.2. Let $d=0$, and $\ell \in \mathcal{R}_{0}$ such that $\int^{\infty} \ell(s) d s / s=\infty$. We assume (C1) and (C2). Then (2.5) is equivalent to

$$
\Delta(1 / \cdot) \in \Pi_{\ell} \text { with } \ell \text {-index } \pi^{-1}
$$

Both imply

$$
c_{n} \sim n^{-1} \ell(n)\{2 \tilde{\ell}(n)\}^{-1 / 2} \quad(n \rightarrow \infty)
$$

If we further assume (A1), then all imply

$$
a_{n} \sim n^{-1} \ell(n)\{2 \tilde{\ell}(n)\}^{-3 / 2} \quad(n \rightarrow \infty) .
$$

Theorem 5.3. Let $-\infty<d \leq 0$ and $\ell \in \mathcal{R}_{0}$. We further assume $\int^{\infty} \ell(s) d s / s<$ $\infty$ if $d=0$. We also assume $(\mathrm{C} 1)$ and $(\mathrm{C} 2)$. Then $(2.5)$ is equivalent to

$$
c_{n} \sim \frac{n^{-(1-2 d)} \ell(n)}{\left\{\sum_{-\infty}^{\infty} \gamma(k)\right\}^{1 / 2}} \quad(n \rightarrow \infty) .
$$

If we further assume (A1), then both imply

$$
a_{n} \sim \frac{n^{-(1-2 d)} \ell(n)}{\left\{\sum_{-\infty}^{\infty} \gamma(k)\right\}^{3 / 2}} \quad(n \rightarrow \infty) .
$$

To prove the theorems above, we start by proving the following lemma which link the asymptotic behaviour of $\left\{c_{n}\right\}$ with that of $\left\{a_{n}\right\}$.

Lemma 5.4. Let $\ell \in \mathcal{R}_{0}$. Let $\left\{u_{n}\right\}_{0}^{\infty}$ and $\left\{v_{n}\right\}_{0}^{\infty}$ be real sequences such that both are eventually decreasing to zero and satisfy the relation

$$
\left(\sum_{n=0}^{\infty} u_{n} z^{n}\right)\left(\sum_{n=0}^{\infty} v_{n} z^{n}\right)=-1 \quad(|z|<1)
$$

(1) Let $0<d<1$. Suppose either $\sum_{0}^{\infty} u_{n}=\infty$ or $\sum_{0}^{\infty} v_{n}=0$. Then the following are equivalent:

$$
\begin{aligned}
& u_{n} \sim n^{-(1-d)} \ell(n) \quad(n \rightarrow \infty), \\
& v_{n} \sim \frac{n^{-(d+1)}}{\ell(n)} \cdot \frac{d \sin (\pi d)}{\pi} \quad(n \rightarrow \infty) .
\end{aligned}
$$

(2) We assume $\int^{\infty} \ell(s) d s / s=\infty$. Suppose either $\sum_{0}^{\infty} u_{n}=\infty$ or $\sum_{0}^{\infty} v_{n}=0$. Then the following are equivalent:

$$
\begin{array}{ll}
u_{n} \sim n^{-1} \ell(n) & (n \rightarrow \infty), \\
v_{n} \sim n^{-1} \frac{\ell(n)}{\tilde{\ell}(n)^{2}} \quad(n \rightarrow \infty) .
\end{array}
$$


(3) Let $1 \leq p<\infty$. Suppose that $\sum_{0}^{\infty} u_{n}$ is finite and nonzero. Then the following are equivalent:

$$
\begin{aligned}
& u_{n} \sim n^{-p} \ell(n) \quad(n \rightarrow \infty), \\
& v_{n} \sim \frac{n^{-p} \ell(n)}{\left(\sum_{0}^{\infty} u_{k}\right)^{2}} \quad(n \rightarrow \infty) .
\end{aligned}
$$

Proof. (1) By assumption, $\sum_{0}^{\infty} u_{n}=\infty$ if and only if $\sum_{0}^{\infty} v_{n}=0$. We set $w_{n}:=\sum_{k=n+1}^{\infty} v_{k}$ for $n \geq 0$. Then

$$
\sum_{n=0}^{\infty} v_{n} z^{n}=(z-1) \sum_{n=0}^{\infty} w_{n} z^{n}
$$

and so

$$
(1-z)\left(\sum_{n=1}^{\infty} u_{n} z^{n}\right)\left(\sum_{n=1}^{\infty} w_{n} z^{n}\right)=1 \quad(|z|<1)
$$

By the monotone density theorem $([\mathrm{BGT}, \S 1.7]),(5.15)$ holds if and only if

$$
w_{n} \sim \frac{n^{-d}}{\ell(n)} \cdot \frac{\sin (\pi d)}{\pi} \quad(n \rightarrow \infty)
$$

which, by Karamata's Tauberian theorem for power series ([BGT, Corollary 1.7.3]), is equivalent to

$$
\sum_{n=0}^{\infty} w_{n} s^{n} \sim \frac{1}{(1-s)^{1-d} \ell(1 /(1-s)) \Gamma(d)} \quad(s \uparrow 1) .
$$

Now by (5.21) this is equivalent to

$$
\sum_{n=0}^{\infty} u_{n} s^{n} \sim(1-s)^{-d} \ell(1 /(1-s)) \Gamma(d) \quad(s \uparrow 1),
$$

which, again by [BGT, Corollary 1.7.3], is equivalent to (5.14).

(2) We set $U(x):=\sum_{n=0}^{[x]} u_{n}$ for $x \geq 0$ and $:=0$ for $x<0$. Here [.] denotes the integer part. We write $\hat{U}$ for the Laplace-Stieltjes transform of $U$ :

$$
\hat{U}(x):=\int_{[0, \infty)} e^{-t x} d U(t)=\sum_{n=0}^{\infty} u_{n} e^{-n x} \quad(x>0) .
$$

Similarly we put $V(x):=\sum_{n=0}^{[x]} v_{n}$ for $x \geq 0$ and $\hat{V}(x):=\sum_{n=0}^{\infty} v_{n} e^{-n x}$ for $x>0$.

First we assume (5.16). Then $\hat{U}(1 / \cdot) \in \Pi_{\ell}$ with $\ell$-index 1 , by de Haan's theorem (see [I5, Theorems 2.3 and 2.4]). On the other hand, since $U(x) \sim$ $\tilde{\ell}(x)$ as $x \rightarrow \infty$, Karamata's Tauberian theorem ([BGT, Theorem 1.7.1]) gives $\hat{U}(1 / x) \sim \tilde{\ell}(x)$ as $x \rightarrow \infty$. We put $\ell_{1}(x):=\ell(x) / \tilde{\ell}(x)^{2}$. Then, by (5.13), for 
$\lambda>0$,

$$
\begin{aligned}
\frac{\hat{V}(1 / \lambda x)-\hat{V}(1 / x)}{\ell_{1}(x)} & =\frac{\hat{U}(1 / \lambda x)-\hat{U}(1 / x)}{\ell(x)} \cdot \frac{\tilde{\ell}(\lambda x)}{\hat{U}(1 / \lambda x)} \cdot \frac{\tilde{\ell}(x)}{\hat{U}(1 / x)} \cdot \frac{\tilde{\ell}(x)}{\tilde{\ell}(\lambda x)} \\
& \rightarrow \log \lambda \quad(x \rightarrow \infty) .
\end{aligned}
$$

Therefore, we see that $\hat{V}(1 / \cdot) \in \Pi_{\ell_{1}}$ with $\ell_{1}$-index 1 , which, by de Haan's Tauberian theorem, implies (5.17).

Next we assume (5.17). Let $w_{n}$ be as in (1). We write, as above, $W(x)=$ $\sum_{n=0}^{[x]} w_{n}$ for $x \geq 0$, and $\hat{W}(x)=\sum_{n=0}^{\infty} w_{n} e^{-n x}$ for $x>0$. Since

$$
w_{n} \sim \int_{n}^{\infty} \ell_{1}(t) d t / t=1 / \tilde{\ell}(n) \quad(n \rightarrow \infty),
$$

we see that $W(x) \sim x / \ell(x)$ as $x \rightarrow \infty$. By Karamata's Tauberian theorem, $\hat{W}(1 / x) \sim x / \tilde{\ell}(x)$ as $x \rightarrow \infty$, so that, by (5.21), $\hat{V}(1 / x) \sim 1 / \tilde{\ell}(x)$ as $x \rightarrow \infty$. On the other hand, (5.17) implies $\hat{V}(1 / \cdot) \in \Pi_{\ell_{1}}$ with $\ell_{1}$-index 1 . Therefore, from an argument similar to the above, it follows that $\hat{U}(1 / \cdot) \in \Pi_{\ell}$ with $\ell$-index 1 , and so (5.16).

(3) We use an argument similar to that of the proof of [I1, Theorem 4.1]. Since $\sum_{0}^{\infty} v_{n}$ is also finite and nonzero, by symmetry it is enough to prove (5.18) $\Rightarrow$ (5.19) only. Set $f(x):=\sum_{n=0}^{\infty} u_{n} e^{-n x}$ for $x>0$. Then from (5.13) we obtain

$$
\sum_{n=0}^{\infty} v_{n} e^{-n x}=-1 / f(x) \quad(x>0) .
$$

Let $r:=[p]$ be the integer part of $p$. By differentiating both sides of the above $r$ times with respect to $x$, we obtain

$$
\sum_{n=1}^{\infty} v_{n} n^{r} e^{-n x}=\frac{\sum_{n=1}^{\infty} u_{n} n^{r} e^{-n x}}{f(x)^{2}}+\frac{F_{r}(x)}{f(x)^{r+1}},
$$

where $F_{r}$ is a polynomial in $\left\{f^{(m)}: m=0,1, \ldots, r-1\right\}$ (see [I1, Lemma 3.3]). Since $r-p>-1$ and

$$
u_{n} n^{r} \sim n^{r-p} \ell(n) \quad(n \rightarrow \infty),
$$

it follows that

$$
\sum_{n=0}^{\infty} u_{n} n^{r} e^{-n x} \sim x^{p-r-1} \ell(1 / x) \Gamma(r-p+1) \quad(x \rightarrow 0+)
$$

(see [I2, Theorem 5.3]). On the other hand, for any $\epsilon>0$ and $0 \leq m \leq r-1$, we have

$$
x^{\epsilon} f^{(m)}(x) \rightarrow \underset{20}{0} \quad(x \rightarrow 0+)
$$


(cf. [I2, Lemma 5.5]); and so

$$
F_{r}(x) /\left\{x^{p-r-1} \ell(1 / x)\right\} \rightarrow 0 \quad(x \rightarrow 0+) .
$$

Thus

$$
\sum_{n=1}^{\infty} v_{n} n^{r} e^{-n x} \sim x^{p-r-1} \ell(1 / x) \frac{\Gamma(r-p+1)}{\left(\sum_{0}^{\infty} u_{k}\right)^{2}} \quad(x \rightarrow 0+) .
$$

Since the sequence $\left\{\log \left(n^{r} v_{n}\right)\right\}$ is slowly increasing ([BGT, §1.7.6]), it follows from Karamata's Tauberian theorem that

$$
v_{n} n^{r} \sim n^{r-p} \ell(n) \quad(n \rightarrow \infty)
$$

(see [I2, Theorem 5.3]). This yields (5.19).

Proof of Theorem 5.1. We use an argument similar to that of the proof of [I6, Theorem 4.1]. The implication $(2.5) \Leftrightarrow(5.5)$ follows from the Abel-Tauber theorem for Fourier cosine series (see [BGT, Corollary 4.10.2]). If we put $g(t):=c_{[t]}$ for $t \geq 0$, then $\gamma(n)=\int_{0}^{\infty} g(t+n) g(t) d t$ for $n \in \mathbb{N}$, and so, by [I6, Proposition 4.3], (5.6) implies (2.5). We put

$$
f(t):=\Delta(2 \arctan t), \quad x(r):=\frac{1-r}{1+r} .
$$

Then, by the change of variable $\theta=2 \arctan t$, we have

$$
\begin{gathered}
\int_{-\infty}^{\infty} \frac{|\log f(t)|}{1+t^{2}} d t=\frac{1}{2} \int_{-\pi}^{\pi}|\log \Delta(\theta)| d \theta<\infty, \\
\int_{-\pi}^{\pi} P_{r}(\theta) \log \Delta(\theta) d \theta=\int_{-\infty}^{\infty} \frac{2 x(r)}{x(r)^{2}+t^{2}} \log f(t) d t \quad(-1<r<1) .
\end{gathered}
$$

Since (5.5) implies

$$
f(t) \sim t^{-2 d} \ell(1 / t) \cdot \frac{1}{2^{2 d+1} \Gamma(1-2 d) \sin (\pi d)} \quad(t \rightarrow 0+),
$$

it follows from [I6, Theorem 4.4] that

$$
\begin{aligned}
\sum_{n=0}^{\infty} c_{n} r^{n} & =(2 \pi)^{1 / 2} \exp \left\{\frac{1}{2 \pi} \int_{-\infty}^{\infty} \frac{2 x(r)}{x(r)^{2}+t^{2}} \log f(t) d t\right\} \sim\{2 \pi f(x(r))\}^{1 / 2} \\
& \sim(1-r)^{-d} \ell(1 /(1-r))^{1 / 2}\left\{\frac{\pi}{\Gamma(1-2 d) \sin (\pi d)}\right\}^{1 / 2} \quad(r \uparrow 1) .
\end{aligned}
$$

Hence (5.6) follows from [BGT, Corollary 1.7.3]. Finally, Lemma 5.4(1) gives the implication $(5.6) \Rightarrow(5.7)$. 
We use the following lemma in the proof of Theorem 5.2 below.

Lemma 5.5. Let c be a positive constant, and let $\ell$ be a slowly varying function such that $\int^{\infty} \ell(s) d s / s=\infty$. For a positive, even, and measurable function $g$ on $\mathbb{R}$ such that

$$
\int_{-\infty}^{\infty} \frac{|\log g(t)|}{1+t^{2}} d t<\infty
$$

we set

$$
K(x):=\exp \left\{\frac{1}{2 \pi} \int_{-\infty}^{\infty} \frac{x}{x^{2}+t^{2}} \log g(t) d t\right\} \quad(x>0) .
$$

Then $g \in \Pi_{\ell}$ with $\ell$-index $c$ implies $K \in \Pi_{\ell_{1}}$ with $\ell_{1}$-index $\sqrt{c} / 2$, where $\ell_{1}(\cdot)$ is defined by $\ell_{1}(t):=\ell(t) / \tilde{\ell}(t)^{1 / 2}$.

Compare the proof below with that of [I6, Theorem 5.2].

Proof. In view of de Haan's theorem ([BGT, Theorem 4.4]), we have

$$
g(t) \sim c \tilde{\ell}(t) \quad(t \rightarrow \infty)
$$

(see the argument in [BGT, p. 164]). Since

$$
K(1 / x)=\exp \left\{\frac{1}{2 \pi} \int_{-\infty}^{\infty} \frac{x}{x^{2}+t^{2}} \log g(1 / t) d t\right\} \quad(x>0),
$$

it follows from [I6, Theorem 4.4] that

$$
K(x) \sim\{c \tilde{\ell}(x)\}^{1 / 2} \quad(x \rightarrow \infty)
$$

We note that $K(x)=\exp A(x)$, where

$$
A(x):=\frac{1}{2 \pi} \int_{-\infty}^{\infty} \frac{1}{1+t^{2}} \log g(t x) d t \quad(x>0) .
$$

Let $\lambda>1$. Then, by the mean value theorem, we have

$$
K(\lambda x)-K(x)=\{A(\lambda x)-A(x)\} \exp B_{\lambda}(x),
$$

where $B_{\lambda}(x)$ is between $A(\lambda x)$ and $A(x)$. Since, by (5.26), both $K(\lambda x) / \tilde{\ell}(x)^{1 / 2}$ and $K(x) / \tilde{\ell}(x)^{1 / 2}$ tend to $\sqrt{c}$ as $x \rightarrow \infty$, we see that

$$
\exp B_{\lambda}(x) \sim\{c \tilde{\ell}(x)\}^{1 / 2} \quad(x \rightarrow \infty) .
$$

Again, by the mean value theorem, we have

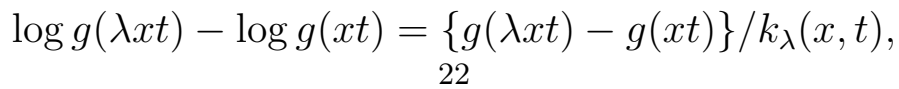


where $k_{\lambda}(x, t)$ is between $g(\lambda x t)$ and $g(x t)$. By (5.25), both $g(x) / g(\lambda x t)$ and $g(x) / g(x t)$ tend to 1 as $x \rightarrow \infty$, whence

$$
g(x) / k_{\lambda}(x, t) \rightarrow 1 \quad(x \rightarrow \infty) \text { for all } t>0
$$

We note that, by [BGT, Theorems 1.5.6 and 3.8.6] (Potter-type bounds), there exist positive constants $D$ and $M$ such that

$$
\begin{aligned}
& |g(\lambda x)-g(x)| / \ell(x) \leq D \lambda \quad(x \geq M), \\
& \ell(y) / \ell(x) \leq D \max \left((y / x)^{1 / 4},(y / x)^{-1 / 4}\right) \quad(x \geq M, y \geq M) \text {, } \\
& g(x) / g(y) \leq D \max \left((y / x)^{1 / 4},(y / x)^{-1 / 4}\right) \quad(x \geq M, y \geq M) .
\end{aligned}
$$

Now we have

$$
\frac{A(\lambda x)-A(x)}{\ell(x) / \tilde{\ell}(x)}=\mathrm{I}(x)-\operatorname{II}(x)+\operatorname{III}(x),
$$

where

$$
\begin{aligned}
\mathrm{I}(x) & :=\frac{\tilde{\ell}(x)}{\pi \ell(x)} \int_{0}^{M} \frac{x}{x^{2}+u^{2}} \log g(\lambda u) d u, \\
\mathrm{II}(x) & :=\frac{\tilde{\ell}(x)}{\pi \ell(x)} \int_{0}^{M} \frac{x}{x^{2}+u^{2}} \log g(u) d u, \\
\operatorname{III}(x) & :=\frac{\tilde{\ell}(x)}{\pi g(x)} \int_{0}^{\infty} F_{\lambda}(x, t) d t
\end{aligned}
$$

with

$$
F_{\lambda}(x, t):=I_{(M / x, \infty)}(t) \cdot \frac{1}{1+t^{2}} \cdot \frac{\{g(\lambda x t)-g(x t)\}}{\ell(x t)} \cdot \frac{\ell(x t)}{\ell(x)} \cdot \frac{g(x)}{k_{\lambda}(x, t)} .
$$

By (5.28), $F_{\lambda}(x, t)$ tends to $c\left(1+t^{2}\right)^{-1} \log \lambda$ as $x \rightarrow \infty$ for all $t>0$. On the other hand, we have, for $x \geq M$,

$$
\begin{aligned}
I_{(M / x, \infty)}(t) \frac{g(x)}{k_{\lambda}(x, t)} & \leq I_{(M / x, \infty)}(t) \max \left(\frac{g(x)}{g(\lambda x t)}, \frac{g(x)}{g(x t)}\right) \\
& \leq D \lambda^{1 / 4} \max \left(t^{1 / 4}, t^{-1 / 4}\right),
\end{aligned}
$$

whence, for $x \geq M$ and $t>0$,

$$
\left|F_{\lambda}(x, t)\right| \leq D^{3} \lambda^{5 / 4} \frac{\max \left(t^{1 / 2}, t^{-1 / 2}\right)}{1+t^{2}} .
$$

Therefore, applying the dominated convergence theorem, we obtain

$$
\operatorname{III}(x) \rightarrow \frac{1}{\pi} \int_{0}^{\infty} \frac{1}{1+t^{2}} d t \cdot \log \lambda=\frac{\log }{2} \lambda \quad(x \rightarrow \infty) .
$$

As for $\mathrm{I}(x)$, we have

$$
|\mathrm{I}(x)| \leq \frac{\tilde{\ell}(x)}{\pi x \ell(x)} \int_{0}^{M}|\log g(\lambda t)| d t \rightarrow 0 \quad(x \rightarrow \infty) .
$$


Similarly, $\mathrm{II}(x) \rightarrow 0$ as $x \rightarrow \infty$. Thus

$$
\frac{A(\lambda x)-A(x)}{\ell(x) / \tilde{\ell}(x)} \rightarrow \frac{\log \lambda}{2} \quad(x \rightarrow \infty) .
$$

Combining this with $(5.27)$, we obtain

$$
\frac{K(\lambda x)-K(x)}{\ell(x) / \tilde{\ell}(x)^{1 / 2}} \rightarrow \frac{\sqrt{c}}{2} \log \lambda \quad(x \rightarrow \infty) .
$$

This proves the lemma.

Proof of Theorem 5.2. By [I4, Theorem 1.3], (2.5) and (5.8) are equivalent. Define $f$ by $(5.22)$, and $g$ by $g(t):=f(1 / t)$. Then, by (5.23) and (5.24),

$$
\begin{gathered}
\int_{-\infty}^{\infty} \frac{|\log g(t)|}{1+t^{2}} d t<\infty, \\
\sum_{n=0}^{\infty} c_{n}\left(\frac{x-1}{x+1}\right)^{n}=(2 \pi)^{1 / 2} \exp \left\{\frac{1}{2 \pi} \int_{-\infty}^{\infty} \frac{x}{x^{2}+t^{2}} \log g(t) d t\right\} \quad(x>1) .
\end{gathered}
$$

Now we note that (5.8) implies $g \in \prod_{\ell}$ with $\ell$-index $\pi^{-1}$ (see [I5, Proposition 2.7]). Hence, it follows from Lemma 5.5 and [I5, Proposition 2.6] that $\sum_{n=0}^{\infty} c_{n} e^{-n / x} \in$ $\Pi_{\ell_{1}}$ with $\ell_{1}$-index $1 / \sqrt{2}$, where $\ell_{1}(x):=\ell(x) /\{\tilde{\ell}(x)\}^{1 / 2}$. Therefore, applying de Haan's Tauberian theorem (cf. [I5, Theorems 2.3 and 2.4]), we obtain (5.9). To complete the proof, we note that, for $C$ large enough,

$$
\int_{C}^{x} \frac{\ell(s)}{\{2 \tilde{\ell}(s)\}^{1 / 2} s} d s=\{2 \tilde{\ell}(t)\}^{1 / 2}-\{2 \tilde{\ell}(C)\}^{1 / 2} \sim\{2 \tilde{\ell}(x)\}^{1 / 2} \quad(x \rightarrow \infty) .
$$

Then, by Lemma 5.4(2), (5.9) implies (5.10).

Proof of Theorem 5.3. From (5.3), it follows that

$$
\sum_{n=-\infty}^{\infty} \gamma(n)=2 \sum_{n=0}^{\infty} \sum_{m=0}^{\infty} c_{n+m} c_{m}-\sum_{m=0}^{\infty}\left(c_{m}\right)^{2}=\left(\sum_{m=0}^{\infty} c_{m}\right)^{2} .
$$

By [I2, Lemma 5.7], $\gamma(n) \sim c_{n}\left(\sum_{m=0}^{\infty} c_{m}\right)$ as $n \rightarrow \infty$, whence (2.5) and (5.11) are equivalent. On the other hand, by Lemma 5.4 (3), (5.11) implies (5.12).

\section{Proof of the MAIN THEOREM}

In this section we prove the main theorem (Theorem 2.1). To do that, we first give the asymptotic behaviour of $\epsilon(n)$ as $n \rightarrow \infty$ (recall $\epsilon(\cdot)$ from $(4.11)$ ). We carry out this first for the long-memory processes, next for the boundary case, and finally for the intermediate-memory processes. It turns out that the two 
infinite sums $\sum_{k=1}^{\infty}$ in (4.3) with $Y=X_{1}$ are negligible as $n \rightarrow \infty$ in the second and third cases but not so in the first case. As a result, the proof for the first case is much more difficult than the others.

First we consider the long-memory processes. For $0<d<\frac{1}{2}$, we put

$$
\begin{aligned}
& A_{1}:=\left(\frac{d}{\pi}\right)^{2} \int_{0}^{\infty}\left\{\int_{0}^{\infty} \frac{d v_{1}}{\left(v_{1}\right)^{1-d}\left(v_{1}+1+u\right)^{1+d}}\right\}^{2} d u \\
& A_{2}:=\left(\frac{d}{\pi}\right)^{4} \int_{0}^{\infty}\left\{\int_{0}^{\infty} \frac{d v_{2}}{\left(v_{2}\right)^{1-d}} \int_{0}^{\infty} \frac{d v_{1}}{\left(v_{1}\right)^{1-d}}\right. \\
&\left.\qquad \int_{0}^{\infty} \frac{d s_{1}}{\left(v_{2}+s_{1}+1+u\right)^{1+d}\left(v_{1}+s_{1}+1\right)^{1+d}}\right\}^{2} d u
\end{aligned}
$$

and for $k \geq 3$,

$$
\begin{aligned}
A_{k}:= & \left(\frac{d}{\pi}\right)^{2 k} \int_{0}^{\infty} d u\left\{\int_{0}^{\infty} \frac{d v_{k}}{\left(v_{k}\right)^{1-d}} \int_{0}^{\infty} \frac{d v_{k-1}}{\left(v_{k-1}\right)^{1-d}} \cdots \int_{0}^{\infty} \frac{d v_{1}}{\left(v_{1}\right)^{1-d}}\right. \\
& \int_{0}^{\infty} \frac{d s_{k-1}}{\left(v_{k}+s_{k-1}+1+u\right)^{1+d}} \int_{0}^{\infty} \frac{d s_{k-2}}{\left(v_{k-1}+s_{k-1}+s_{k-2}+1\right)^{1+d}} \\
& \left.\ldots \int_{0}^{\infty} \frac{d s_{2}}{\left(v_{3}+s_{3}+s_{2}+1\right)^{1+d}} \int_{0}^{\infty} \frac{d s_{1}}{\left(v_{2}+s_{2}+s_{1}+1\right)^{1+d}\left(v_{1}+s_{1}+1\right)^{1+d}}\right\}^{2} .
\end{aligned}
$$

By the equality

$$
\int_{0}^{\infty} \frac{d v}{(x+v)^{1+d} v^{1-d}}=\frac{1}{x d} \quad(0<x<\infty)
$$

$A_{k}$ may be expressed more simply as follows: $A_{1}=\pi^{-2}$,

$$
A_{2}=\pi^{-4} \int_{0}^{\infty}\left\{\int_{0}^{\infty} \frac{d s_{1}}{\left(s_{1}+1+u\right)\left(s_{1}+1\right)}\right\}^{2} d u
$$

and for $k \geq 3$,

$$
\begin{aligned}
& A_{k}=\pi^{-2 k} \int_{0}^{\infty}\left\{\int_{0}^{\infty} \frac{d s_{k-1}}{\left(s_{k-1}+1+u\right)} \int_{0}^{\infty} \frac{d s_{k-2}}{\left(s_{k-1}+s_{k-2}+1\right)}\right. \\
&\left.\ldots \int_{0}^{\infty} \frac{d s_{2}}{\left(s_{3}+s_{2}+1\right)} \int_{0}^{\infty} \frac{d s_{1}}{\left(s_{2}+s_{1}+1\right)\left(s_{1}+1\right)}\right\}^{2} d u .
\end{aligned}
$$

In particular, we see that $A_{k}$ does not depend on $d$. The value of $A_{k}$ for $k \geq 2$ will be identified by (6.15) below.

Recall $d_{k}(n, p)$ from Theorems 4.5 and 4.6 .

Proposition 6.1. Let $0<d<\frac{1}{2}$ and $\ell \in \mathcal{R}_{0}$. We assume $(\mathrm{C} 1)$, (C2), and (A1).

Then, for $k \in \mathbb{N},(2.5)$ implies

$$
\sum_{p=0}^{\infty}\left\{d_{k}(n, p)\right\}^{2} \sim n^{-1} A_{k} \sin ^{2 k}(\pi d) \quad(n \rightarrow \infty) .
$$


Proof. We give full details for the case $k=2$, and then describe how to adapt the argument to the general case.

Choose $\delta$ from the interval $\left(0, \min \left(d, \frac{1}{14}\right)\right)$. Then, by Theorem 5.1 and Potter's bound ([BGT, Theorem 1.5.6]), there exists $M>0$ such that

$$
\begin{gathered}
0 \leq \frac{a_{n}}{a_{m}} \leq \frac{2}{\{(n+3) / m\}^{1+d-\delta}} \quad(n \geq m \geq M) \\
0 \leq \frac{c_{n}}{c_{m}} \leq 2 \max \left(\left(\frac{m}{n+1}\right)^{1-d+\delta},\left(\frac{m}{n+1}\right)^{1-d-\delta}\right) \quad(n \geq M, m \geq M) .
\end{gathered}
$$

We note that we choose $n+3$ in (6.3) and $n+1$ in (6.4) instead of $n$ because these are more useful for our purpose. We set, for $x>0, g_{0}(x):=I_{(0, M)}(x) c_{[x]}$, $g_{1}(x):=I_{[M, \infty)}(x) c_{[x]}$, and

$$
G(x):=2 \max \left(x^{-(1-d+\delta)}, x^{-(1-d-\delta)}\right) .
$$

Here $[\cdot]$ denotes integer part as before. Then we have

$$
\begin{gathered}
0 \leq \frac{g_{1}(n v)}{c_{n}} \leq G(v) \quad(n \geq M, v>0), \\
\frac{g_{1}(n v)}{c_{n}} \rightarrow v^{-(1-d)} \quad(n \rightarrow \infty) \quad(v>0) .
\end{gathered}
$$

From Theorem 4.6, it follows that, for $n$ large enough,

$$
\begin{aligned}
& \sum_{p=0}^{\infty}\left\{d_{2}(n-2, p)\right\}^{2} \\
& =\int_{0}^{\infty}\left\{\int_{0}^{\infty} d v_{2} c_{\left[v_{2}\right]} \int_{0}^{\infty} d v_{1} c_{\left[v_{1}\right]} \int_{0}^{\infty} a_{\left[v_{2}\right]+\left[s_{1}\right]+n+[u]} a_{\left[v_{1}\right]+\left[s_{1}\right]+n} d s_{1}\right\}^{2} d u \\
& =n^{7}\left\{a_{n} c_{n}\right\}^{4} \int_{0}^{\infty}\left\{\int_{0}^{\infty} d v_{2} \frac{c_{\left[n v_{2}\right]}}{c_{n}} \int_{0}^{\infty} d v_{1} \frac{c_{\left[n v_{1}\right]}}{c_{n}}\right. \\
& \left.\qquad \int_{0}^{\infty} \frac{a_{\left[n v_{2}\right]+\left[n s_{1}\right]+n+[n u]}}{a_{n}} \cdot \frac{a_{\left[n v_{1}\right]+\left[n s_{1}\right]+n}}{a_{n}} d s_{1}\right\}^{2} d u \\
& =n^{7}\left\{a_{n} c_{n}\right\}^{4} \cdot\left\|\mathrm{I}_{00}(n)+\mathrm{I}_{01}(n)+\mathrm{I}_{10}(n)+\mathrm{I}_{11}(n)\right\|^{2},
\end{aligned}
$$

where $\|\cdot\|$ is the norm of $L^{2}((0, \infty), d u)$ and $\mathrm{I}_{i j}(n)=\mathrm{I}_{i j}(n)(u)$ are defined by

$$
\begin{aligned}
\mathrm{I}_{i j}(n):=\int_{0}^{\infty} d v_{2} \frac{g_{i}\left(n v_{2}\right)}{c_{n}} \int_{0}^{\infty} d v_{1} \frac{g_{j}\left(n v_{1}\right)}{c_{n}} \\
\quad \int_{0}^{\infty} \frac{a_{\left[n v_{2}\right]+\left[n s_{1}\right]+n+[n u]}}{a_{n}} \cdot \frac{a_{\left[n v_{1}\right]+\left[n s_{1}\right]+n}}{a_{n}} d s_{1} .
\end{aligned}
$$

First we consider $\mathrm{I}_{11}(n)$. Since

$$
v_{2}+s_{1}+1-\frac{3}{n}+u<\frac{\left[n v_{2}\right]+\left[n s_{1}\right]+n+[n u]}{{ }_{26}^{n}} \leq v_{2}+s_{1}+1+u,
$$


we have

$$
\begin{aligned}
& 0 \leq \frac{a_{\left[n v_{2}\right]+\left[n s_{1}\right]+n+[n u]}}{a_{n}} \leq 2\left(v_{2}+s_{1}+1+u\right)^{-(1+d-\delta)} \\
& \quad\left(n \geq M, v_{2}>0, s_{1}>0, u>0\right), \\
& \frac{a_{\left[n v_{2}\right]+\left[n s_{1}\right]+n+[n u]}}{a_{n}} \rightarrow\left(v_{2}+s_{1}+u+1\right)^{-(1+d)} \quad(n \rightarrow \infty) .
\end{aligned}
$$

Similarly

$$
\begin{gathered}
0 \leq \frac{a_{\left[n v_{1}\right]+\left[n s_{1}\right]+n}}{a_{n}} \leq 2\left(v_{1}+s_{1}+1\right)^{-(1+d-\delta)} \quad\left(n \geq M, v_{1}>0, s_{1}>0\right), \\
\frac{a_{\left[n v_{1}\right]+\left[n s_{1}\right]+n}}{a_{n}} \rightarrow\left(v_{2}+s_{1}+1\right)^{-(1+d)} \quad(n \rightarrow \infty) .
\end{gathered}
$$

Now

$$
\begin{aligned}
\left(v_{2}\right. & \left.+s_{1}+u+1\right)^{-(1+d-\delta)}\left(v_{1}+s_{1}+1\right)^{-(1+d-\delta)} \\
& =\left(v_{2}+s_{1}+u+1\right)^{-(d+2 \delta)-4 \delta-(1-7 \delta)}\left(v_{1}+s_{1}+1\right)^{-(d+2 \delta)-(1-3 \delta)} \\
& \leq\left(v_{2}+1\right)^{-(d+2 \delta)}\left(v_{1}+1\right)^{-(d+2 \delta)}\left(s_{1}+1\right)^{-(1+\delta)}(u+1)^{-(1-7 \delta)}
\end{aligned}
$$

and so, for $n \geq M$, we have

$$
\begin{aligned}
\left|\mathrm{I}_{11}(n)\right|^{2} & \leq \frac{2^{4}}{(1+u)^{2-14 \delta}} \\
& \times\left\{\int_{0}^{\infty} \frac{G\left(v_{2}\right)}{\left(1+v_{2}\right)^{d+2 \delta}} d v_{2} \cdot \int_{0}^{\infty} \frac{G\left(v_{1}\right)}{\left(1+v_{1}\right)^{d+2 \delta}} d v_{1} \cdot \int_{0}^{\infty} \frac{1}{\left(1+s_{1}\right)^{1+\delta}} d s_{1}\right\}^{2} .
\end{aligned}
$$

Hence, applying the dominated convergence theorem twice, we obtain

$$
\left\|\mathrm{I}_{11}(n)\right\|^{2} \rightarrow(\pi / d)^{4} A_{2} \quad(n \rightarrow \infty)
$$

Next we consider the remaining integrals. From the above estimates, we have, for $n \geq M$,

$$
\begin{aligned}
\left|\mathrm{I}_{01}(n)\right|^{2} & \leq \frac{1}{\left(n c_{n}\right)^{2}} \cdot \frac{2^{4}}{(1+u)^{2-14 \delta}} \\
& \times\left\{\int_{0}^{M}\left|g_{0}\left(v_{2}\right)\right| d v_{2} \cdot \int_{0}^{\infty} \frac{G\left(v_{1}\right)}{\left(1+v_{1}\right)^{d+2 \delta}} d v_{1} \cdot \int_{0}^{\infty} \frac{1}{\left(1+s_{1}\right)^{1+\delta}} d s_{1}\right\}^{2}
\end{aligned}
$$

so that $\left\|\mathrm{I}_{01}(n)\right\| \rightarrow 0$ as $n \rightarrow \infty$. Similarly, $\left\|\mathrm{I}_{10}(n)\right\| \rightarrow 0$ and $\left\|\mathrm{I}_{00}(n)\right\| \rightarrow 0$ as $n \rightarrow \infty$.

Combining, we have

$$
\left\|\mathrm{I}_{00}(n)+\mathrm{I}_{01}(n)+\mathrm{I}_{10}(n)+\mathrm{I}_{11}(n)\right\|^{2} \rightarrow(\pi / d)^{4} A_{2} \quad(n \rightarrow \infty) .
$$

However, by Theorem 5.1,

$$
n^{7}\left\{a_{n} c_{n}\right\}^{4} \sim\left\{\frac{d \sin (\pi d)}{\pi}\right\}_{27}^{4} n^{-1} \quad(n \rightarrow \infty)
$$


whence, as asserted in (6.2),

$$
\sum_{p=0}^{\infty}\left\{d_{2}(n, p)\right\}^{2} \sim n^{-1} A_{2} \sin ^{4}(\pi d) \quad(n \rightarrow \infty) .
$$

In the general case $k \geq 1$, we choose $\delta$ from the interval $(0, \min \{d, 1 /(8 k-2)\})$, and instead of (6.6) we use the following estimate:

$$
\begin{aligned}
&\left(v_{k}+s_{k-1}+u+1\right)^{-(1+d-\delta)}\left(v_{k-1}+s_{k-1}+s_{k-2}+1\right)^{-(1+d-\delta)} \\
& \quad \cdots\left(v_{2}+s_{2}+s_{1}+1\right)^{-(1+d-\delta)}\left(v_{1}+s_{1}+1\right)^{-(1+d-\delta)} \\
&=\left(v_{k}+s_{k-1}+u+1\right)^{-(d+2 \delta)-(4 k-4) \delta-\{1-(4 k-1) \delta\}} \\
& \quad \times\left(v_{k-1}+s_{k-1}+s_{k-2}+1\right)^{-(d+2 \delta)-\{1-(4 k-5) \delta\}-(4 k-8) \delta} \\
& \quad \cdots\left(v_{2}+s_{2}+s_{1}+1\right)^{-(d+2 \delta)-(1-7 \delta)-4 \delta}\left(v_{1}+s_{1}+1\right)^{-(d+2 \delta)-(1-3 \delta)} \\
& \leq\left(v_{k}+1\right)^{-(d+2 \delta)} \cdots\left(v_{1}+1\right)^{-(d+2 \delta)} \\
& \times\left(s_{k-1}+1\right)^{-(1+\delta)} \cdots\left(s_{1}+1\right)^{-(1+\delta)}(u+1)^{-1+(4 k-1) \delta} .
\end{aligned}
$$

Here we note that $\delta<1 /(8 k-2)$ implies

$$
\int_{0}^{\infty} \frac{1}{(u+1)^{2-(8 k-2) \delta}} d u<\infty
$$

Then we can prove the assertion for $k \geq 1$ similarly.

Now, in a manner similar to the definition of $d_{k}(n, p)$, we introduce other infinite sums consisting of $c_{n}$ and $a_{n}$, for later use. Thus, under the assumptions (C1) and (A1), we choose $M \in \mathbb{N}$ so that $a_{n+2} \geq 0$ for all $n \geq M$, and put for $k \geq 2, n \geq M$, and $p \geq 0$,

$$
e_{k}(n, p):=\sum_{m_{1}=1}^{\infty} a_{n+m_{1}+1} \sum_{m_{2}=1}^{\infty} b_{n+m_{2}}^{m_{1}} \ldots \sum_{m_{k-1}=1}^{\infty} b_{n+m_{k-1}}^{m_{k-2}} \sum_{m_{k}=1}^{\infty} b_{n+m_{k}}^{m_{k-1}} c_{m_{k}+p} .
$$

Then, as in Theorem 4.6,

$$
e_{2}(n, p)=\sum_{v_{2}=0}^{\infty} c_{v_{2}+p+1} \sum_{v_{1}=0}^{\infty} c_{v_{1}} \sum_{m_{1}=0}^{\infty} a_{v_{2}+m_{1}+n+2} a_{v_{1}+m_{1}+n+2}
$$

and for $k \geq 3$

$$
\begin{aligned}
& e_{k}(n, p)=\sum_{v_{k}=0}^{\infty} c_{v_{k}+p+1} \sum_{v_{k-1}=0}^{\infty} c_{v_{k-1}} \\
& \ldots \sum_{v_{1}=0}^{\infty} c_{v_{1}} \sum_{m_{k-1}=0}^{\infty} a_{v_{k}+m_{k-1}+n+2} \sum_{m_{k-2}=0}^{\infty} a_{v_{k-1}+m_{k-1}+m_{k-2}+n+2} \\
& \ldots \sum_{m_{2}=0}^{\infty} a_{v_{3}+m_{3}+m_{2}+n+2} \sum_{m_{1}=0}^{\infty} a_{v_{2}+m_{2}+m_{1}+n+2} a_{v_{1}+m_{1}+n+2} . \\
& 28
\end{aligned}
$$


For $0<d<\frac{1}{2}$ and $k \geq 2$, we also define $B_{k}(d)$, in a manner similar to the definition of $A_{k}$, as follows:

$$
\begin{aligned}
B_{2}(d):=\left(\frac{d}{\pi}\right)^{4} \int_{0}^{\infty} & \left\{\int_{0}^{\infty} \frac{d v_{2}}{\left(v_{2}+u\right)^{1-d}} \int_{0}^{\infty} \frac{d v_{1}}{\left(v_{1}\right)^{1-d}}\right. \\
& \left.\int_{0}^{\infty} \frac{d s_{1}}{\left(v_{2}+s_{1}+1\right)^{1+d}\left(v_{1}+s_{1}+1\right)^{1+d}}\right\}^{2} d u,
\end{aligned}
$$

and for $k \geq 3$,

$$
\begin{aligned}
& B_{k}(d):=\left(\frac{d}{\pi}\right)^{2 k} \int_{0}^{\infty} d u\left\{\int_{0}^{\infty} \frac{d v_{k}}{\left(v_{k}+u\right)^{1-d}} \int_{0}^{\infty} \frac{d v_{k-1}}{\left(v_{k-1}\right)^{1-d}} \cdots \int_{0}^{\infty} \frac{d v_{1}}{\left(v_{1}\right)^{1-d}}\right. \\
& \quad \int_{0}^{\infty} \frac{d s_{k-1}}{\left(v_{k}+s_{k-1}+1\right)^{1+d}} \int_{0}^{\infty} \frac{d s_{k-2}}{\left(v_{k-1}+s_{k-1}+s_{k-2}+1\right)^{1+d}} \\
& \left.\quad \ldots \int_{0}^{\infty} \frac{d s_{2}}{\left(v_{3}+s_{3}+s_{2}+1\right)^{1+d}} \int_{0}^{\infty} \frac{d s_{1}}{\left(v_{2}+s_{2}+s_{1}+1\right)^{1+d}\left(v_{1}+s_{1}+1\right)^{1+d}}\right\}^{2} .
\end{aligned}
$$

By the equality $(6.1), B_{k}(d)$ with $k \geq 3$ defined above may be expressed more simply as follows:

$$
\begin{aligned}
& B_{k}(d)=\frac{d^{2}}{\pi^{2 k}} \int_{0}^{\infty} d u\left\{\int_{0}^{\infty} \frac{d v_{k}}{\left(v_{k}+u\right)^{1-d}} \int_{0}^{\infty} \frac{d s_{k-1}}{\left(v_{k}+s_{k-1}+1\right)^{1+d}}\right. \\
& \left.\int_{0}^{\infty} \frac{d s_{k-2}}{\left(s_{k-1}+s_{k-2}+1\right)} \cdots \int_{0}^{\infty} \frac{d s_{2}}{\left(s_{3}+s_{2}+1\right)} \int_{0}^{\infty} \frac{d s_{1}}{\left(s_{2}+s_{1}+1\right)\left(s_{1}+1\right)}\right\}^{2} .
\end{aligned}
$$

The following proposition is an analogue of Proposition 6.1 for $\left\{e_{k}(n, p)\right\}$. We use it, in the proof of Theorem 6.4 below, to estimate the difference between upper and lower bounds.

Proposition 6.2. Let $0<d<\frac{1}{2}$ and $\ell \in \mathcal{R}_{0}$. We assume (C1), (C2), and (A1).

Then, for $k \geq 2$, (2.5) implies

$$
\sum_{p=0}^{\infty}\left\{e_{k}(n, p)\right\}^{2} \sim n^{-1} B_{k}(d) \sin ^{2 k}(\pi d) \quad(n \rightarrow \infty) .
$$

Proof. For $k \geq 3$ and $n$ large enough, we have

$$
\begin{aligned}
& \sum_{p=0}^{\infty}\left\{e_{k}(n-2, p)\right\}^{2}=n^{4 k-1}\left\{a_{n} c_{n}\right\}^{2 k} \\
& \times \int_{0}^{\infty} d u\left\{\int_{0}^{\infty} d v_{k} \frac{c_{\left[n v_{k}\right]+[n u]+1}}{c_{n}} \int_{0}^{\infty} d v_{k-1} \frac{c_{\left[n v_{k-1}\right]}}{c_{n}} \cdots \int_{0}^{\infty} d v_{1} \frac{c_{\left[n v_{1}\right]}}{c_{n}}\right. \\
& \quad \int_{0}^{\infty} d s_{k-1} \frac{a_{\left[n v_{k}\right]+\left[n s_{k-1}\right]+n}}{a_{n}} \int_{0}^{\infty} d s_{k-2} \frac{a_{\left[n v_{k-1}\right]+\left[n s_{k-1}\right]+\left[n s_{k-2}\right]+n}}{a_{n}} \\
& \left.\ldots \int_{0}^{\infty} d s_{2} \frac{a_{\left[n v_{3}\right]+\left[n s_{3}\right]+\left[n s_{2}\right]+n}}{a_{n}} \int_{0}^{\infty} \frac{a_{\left[n v_{2}\right]+\left[n s_{2}\right]+\left[n s_{1}\right]+n}}{a_{n}} \cdot \frac{a_{\left[n v_{1}\right]+\left[n s_{1}\right]+n}}{a_{n}} d s_{1}\right\}^{2} .
\end{aligned}
$$


However, for simplicity, we give details for the case $k=2$ only, and finish the proof with brief notes for the general case.

Take $\delta \in\left(0, \min \left(\frac{1}{2}-d, \frac{1}{5} d\right)\right)$, and $M$ so large that both (6.3) and (6.4) hold. We set $q_{0}(x):=I_{(0, M)}(x)$ and $q_{1}(x):=I_{[M, \infty)}(x)$. Then, for $n$ large enough, we have

$$
\sum_{p=0}^{\infty}\left\{e_{2}(n-2, p)\right\}^{2}=n^{7}\left\{a_{n} c_{n}\right\}^{4} \cdot\left\|\mathrm{I}_{00}(n)+\mathrm{I}_{01}(n)+\mathrm{I}_{10}(n)+\mathrm{I}_{11}(n)\right\|^{2},
$$

where $\|\cdot\|$ is the norm of $L^{2}((0, \infty), d u)$ and $\mathrm{I}_{i j}(n)=\mathrm{I}_{i j}(n)(u)$ are defined by

$$
\begin{aligned}
\mathrm{I}_{i j}(n):=\int_{0}^{\infty} d v_{2} q_{i}([n u]) & \frac{c_{\left[n v_{2}\right]+[n u]+1}}{c_{n}} \int_{0}^{\infty} d v_{1} q_{j}\left(\left[n v_{1}\right]\right) \frac{c_{\left[n v_{1}\right]}}{c_{n}} \\
& \int_{0}^{\infty} \frac{a_{\left[n v_{2}\right]+\left[n s_{1}\right]+n}}{a_{n}} \cdot \frac{a_{\left[n v_{1}\right]+\left[n s_{1}\right]+n}}{a_{n}} d s_{1} .
\end{aligned}
$$

First we consider $\mathrm{I}_{11}(n)$. From

$$
v_{2}+u-\frac{1}{n}<\frac{\left[n v_{2}\right]+[n u]+1}{n} \leq v_{2}+u+\frac{1}{n},
$$

we have

$$
\begin{gathered}
q_{1}([n u]) \cdot \frac{c_{\left[n v_{2}\right]+[n u]+1}}{c_{n}} \leq G\left(v_{2}+u\right) \quad\left(n \geq M, u>0, v_{2}>0\right), \\
q_{1}([n u]) \cdot \frac{c_{\left[n v_{2}\right]+[n u]+1}}{c_{n}} \rightarrow\left(v_{2}+u\right)^{-(1-d)} \quad(n \rightarrow \infty) \quad\left(u>0, v_{2}>0\right),
\end{gathered}
$$

where $G$ is the function defined by (6.5). Since

$$
\begin{aligned}
\left(v_{2}\right. & \left.+s_{1}+1\right)^{-(1+d-\delta)}\left(v_{1}+s_{1}+1\right)^{-(1+d-\delta)} \\
& =\left(v_{2}+s_{1}+1\right)^{-(1+d-5 \delta)-4 \delta}\left(v_{1}+s_{1}+1\right)^{-(d+2 \delta)-(1-3 \delta)} \\
& \leq\left(v_{2}+1\right)^{-(1+d-5 \delta)}\left(v_{1}+1\right)^{-(d+2 \delta)}\left(s_{1}+1\right)^{-(1+\delta)},
\end{aligned}
$$

we have, for $n \geq M$,

$$
\left|I_{11}(n)\right|^{2} \leq 2^{4}\left\{\int_{0}^{\infty} \frac{G\left(u+v_{2}\right) d v_{2}}{\left(v_{2}+1\right)^{1+d-5 \delta}} \cdot \int_{0}^{\infty} \frac{G\left(v_{1}\right) d v_{1}}{\left(v_{1}+1\right)^{d+2 \delta}} \cdot \int_{0}^{\infty} \frac{d s_{1}}{\left(s_{1}+1\right)^{1+\delta}}\right\}^{2} .
$$

Now

$$
G\left(u+v_{2}\right) \leq I_{(0,1)}(u) G\left(v_{2}\right)+I_{[1, \infty)}(u) G(u)
$$

and so, if we notice that $(a+b)^{2} \leq 2\left(a^{2}+b^{2}\right)$, then

$$
\begin{aligned}
& \left|I_{11}(n)\right|^{2} \leq 2^{5}\left\{\int_{0}^{\infty} \frac{G\left(v_{1}\right)}{\left(v_{1}+1\right)^{d+2 \delta}} d v_{1} \cdot \int_{0}^{\infty} \frac{1}{\left(s_{1}+1\right)^{1+\delta}} d s_{1}\right\}^{2} \\
& \times\left[\left\{\int_{0}^{\infty} \frac{I_{(0,1)}(u) G\left(v_{2}\right)}{\left(v_{2}+1\right)^{1+d-5 \delta}} d v_{2}\right\}^{2}+\left\{\int_{0}^{\infty} \frac{I_{[1, \infty)}(u) G(u)}{\left(v_{2}+1\right)^{1+d-5 \delta}} d v_{2}\right\}^{2}\right] .
\end{aligned}
$$


The right-hand side is in $L^{1}((0, \infty), d u)$, whence, by the dominated convergence theorem, we have

$$
\left\|\mathrm{I}_{11}(n)\right\|^{2} \rightarrow(\pi / d)^{4} B_{2}(d) \quad(n \rightarrow \infty)
$$

We turn to the remaining integrals. We have, for $n \geq M$,

$$
\begin{aligned}
& \left|I_{01}(n)\right|^{2} \leq 2^{4} I_{(0,1)}([n u])\left(\frac{\max c_{k}}{c_{n}}\right)^{2} \\
& \times\left\{\int_{0}^{\infty} \frac{d v_{2}}{\left(v_{2}+1\right)^{1+d-5 \delta}} \cdot \int_{0}^{\infty} \frac{G\left(v_{1}\right)}{\left(v_{1}+1\right)^{d+2 \delta}} d v_{1} \cdot \int_{0}^{\infty} \frac{d s_{1}}{\left(s_{1}+1\right)^{1+\delta}}\right\}^{2} .
\end{aligned}
$$

Hence it follows that $\left\|\mathrm{I}_{01}(n)\right\| \rightarrow 0$ as $n \rightarrow \infty$. Furthermore, for $n \geq M,\left|\mathrm{I}_{10}(n)\right|^{2}$ is at most

$$
2^{4}\left(\frac{\max c_{k}}{n c_{n}}\right)^{2}\left\{\int_{0}^{\infty} \frac{G\left(u+v_{2}\right)}{\left(v_{2}+1\right)^{1+d-5 \delta}} d v_{2} \cdot \int_{0}^{M} d v_{1} \cdot \int_{0}^{\infty} \frac{d s_{1}}{\left(s_{1}+1\right)^{1+\delta}}\right\}^{2} .
$$

Hence, by (6.11) and (5.6), $\left\|\mathrm{I}_{10}(n)\right\| \rightarrow 0$ as $n \rightarrow \infty$. Similarly, $\left\|\mathrm{I}_{00}(n)\right\| \rightarrow 0$ as $n \rightarrow \infty$.

Combining, we obtain

$$
\left\|\mathrm{I}_{00}(n)+\mathrm{I}_{01}(n)+\mathrm{I}_{10}(n)+\mathrm{I}_{11}(n)\right\|^{2} \rightarrow(\pi / d)^{4} B_{2}(d) \quad(n \rightarrow \infty),
$$

and so, from (6.7), as asserted,

$$
\sum_{p=0}^{\infty}\left\{e_{2}(n, p)\right\}^{2} \sim n^{-1} B_{2}(d) \sin ^{4}(\pi d) \quad(n \rightarrow \infty) .
$$

As for the general $k \geq 1$, we choose $\delta \in\left(0, \min \left\{\frac{1}{2}-d, d /(4 k-3)\right\}\right)$, and instead of (6.10) we use the estimate

$$
\begin{aligned}
& \left(v_{k}+s_{k-1}+1\right)^{-(1+d-\delta)}\left(v_{k-1}+s_{k-1}+s_{k-2}+1\right)^{-(1+d-\delta)} \\
& \cdots\left(v_{2}+s_{2}+s_{1}+1\right)^{-(1+d-\delta)}\left(v_{1}+s_{1}+1\right)^{-(1+d-\delta)} \\
= & \left(v_{k}+s_{k-1}+1\right)^{-\{1+d-(4 k-3) \delta\}-(4 k-4) \delta} \\
& \times\left(v_{k-1}+s_{k-1}+s_{k-2}+1\right)^{-(d+2 \delta)-\{1-(4 k-5) \delta\}-(4 k-8) \delta} \\
& \cdots\left(v_{2}+s_{2}+s_{1}+1\right)^{-(d+2 \delta)-(1-7 \delta)-4 \delta}\left(v_{1}+s_{1}+1\right)^{-(d+2 \delta)-(1-3 \delta)} \\
\leq & \left(v_{k}+1\right)^{-\{1+d-(4 k-3) \delta\}}\left(v_{k-1}+1\right)^{-(d+2 \delta)} \cdots\left(v_{1}+1\right)^{-(d+2 \delta)} \\
& \times\left(s_{k-1}+1\right)^{-(1+\delta)} \cdots\left(s_{1}+1\right)^{-(1+\delta)} .
\end{aligned}
$$

We note that $\delta<d /(4 k-3)$ implies

$$
\int_{0}^{\infty} \frac{1}{\left(v_{k}+1\right)^{1+d-(4 k-3) \delta}} d v_{k}<\infty
$$


and that $\delta<\frac{1}{2}-d$ implies $\int_{1}^{\infty} G(u)^{2} d u<\infty$. We also have

$$
\int_{0}^{\infty} \frac{G\left(v_{k}\right)}{\left(v_{k}+1\right)^{1+d-(4 k-3) \delta}} d v_{k}<\infty .
$$

In this way, we can prove the general assertion in a manner similar to the above.

For $0<d<\frac{1}{2}$, we define an integral operator $K_{d}$ on $L^{2}((0, \infty), d u)$ by

$$
K_{d} f(x):=\int_{0}^{\infty} k_{d}(x, y) f(y) d y
$$

where

$$
k_{d}(x, y):=\int_{0}^{\infty} \frac{1}{(x+v)^{1-d}(v+y)^{1+d}} d v \quad(x>0, y>0) .
$$

We write $\left\|K_{d}\right\|$ for the operator norm of $K_{d}$.

Lemma 6.3. For $0<d<\frac{1}{2}, K_{d}$ is a bounded operator on $L^{2}((0, \infty)$, du) such that $\left\|K_{d}\right\| \leq(\pi / d) \tan (\pi d)$.

Proof. Since

$$
k_{d}(x, x y)=\frac{1}{x} \int_{0}^{\infty} \frac{1}{(1+s)^{1-d}(s+y)^{1+d}} d s
$$

we have

$$
\begin{aligned}
\int_{0}^{\infty} k_{d}(x, y)(x / y)^{\frac{1}{2}} d y & =x \int_{0}^{\infty} k_{d}(x, x y) y^{-\frac{1}{2}} d y \\
& =\int_{0}^{\infty} \frac{d s}{(s+1)^{1-d} s^{d+\frac{1}{2}}} \cdot \int_{0}^{\infty} \frac{d y}{(y+1)^{1+d} y^{\frac{1}{2}}} \\
& =B\left(\frac{1}{2}, \frac{1}{2}-d\right) B\left(d+\frac{1}{2}, \frac{1}{2}\right)=\frac{\pi}{d} \tan (\pi d) .
\end{aligned}
$$

Similarly

$$
\int_{0}^{\infty} k_{d}(x, y)(y / x)^{\frac{1}{2}} d x=\frac{\pi}{d} \tan (\pi d) .
$$

Thus the lemma follows (see [HLP, Theorem 319]).

Now we are ready to prove the following theorem which gives the asymptotic behaviour of $\epsilon(\cdot)$ for long-memory processes. See [IK], where an analogous result for a special continuous-time stationary process is given.

Theorem 6.4. Let $0<d<\frac{1}{2}$ and $\ell \in \mathcal{R}_{0}$. We assume (C1), (C2), and (A1). Then (2.5) implies

$$
\epsilon(n) \sim \frac{d^{2}}{n} \quad(n \rightarrow \infty)
$$


Proof. By Proposition 4.3, we have $\sum_{0}^{\infty}\left|a_{k}\right|<\infty$. Hence it follows from (4.2) and (4.14)-(4.16) that, for $m \geq 1$,

$$
\begin{aligned}
& \sum_{k=1}^{2 m-1} \sum_{p=0}^{\infty}\left\{d_{k}(n, p)\right\}^{2} \leq \epsilon(n) \\
& \quad=\sum_{k=1}^{2 m-1} \sum_{p=0}^{\infty}\left\{d_{k}(n, p)\right\}^{2}+\left(c_{0}\right)^{-2}\left\|P_{[-n, 0]}^{\perp}\left\{P_{[-n, \infty)} P_{(-\infty, 0]}\right\}^{m} X_{1}\right\|^{2} .
\end{aligned}
$$

Let $n \rightarrow \infty$. Then, by Proposition 6.1,

$$
\begin{aligned}
& \sum_{k=1}^{2 m-1} A_{k} \sin ^{2 k}(\pi d) \leq \liminf _{n \rightarrow \infty} \epsilon(n) n \leq \limsup _{n \rightarrow \infty} \epsilon(n) n \\
& \leq \sum_{k=1}^{2 m-1} A_{k} \sin ^{2 k}(\pi d)+\limsup _{n \rightarrow \infty}\left\{\left(c_{0}\right)^{-2} n\left\|P_{[-n, 0]}^{\perp}\left\{P_{[-n, \infty)} P_{(-\infty, 0]}\right\}^{m} X_{1}\right\|^{2}\right\} .
\end{aligned}
$$

Now it follows from (4.13) that

$$
\left\{P_{[-n, \infty)} P_{(-\infty, 0]}\right\}^{m} X_{1}=c_{0} Z(n, m) \quad\left(\bmod H_{[-n, 0]}\right),
$$

where

$$
Z(n, m):=\sum_{j_{1}=1}^{\infty} a_{n+j_{1}+1} \sum_{j_{2}=1}^{\infty} b_{n+j_{2}}^{j_{1}} \cdots \sum_{j_{2 m}=1}^{\infty} b_{n+j_{2 m}}^{j_{2 m-1}} X_{j_{2 m}} .
$$

Let $\left\{\xi_{k}\right\}$ be the complete orthonormal system for $H$ defined by (4.6). Then

$$
\begin{aligned}
\left(c_{0}\right)^{-2} & \left\|P_{[-n, 0]}^{\perp}\left\{P_{[-n, \infty)} P_{(-\infty, 0]}\right\}^{m} X_{1}\right\|^{2}=\left\|P_{[-n, 0]}^{\perp} Z(n, m)\right\|^{2} \\
\leq & \|Z(n, m)\|^{2}=\sum_{p=-\infty}^{\infty}\left(Z(n, m), \xi_{p}\right)^{2} \\
= & \sum_{p=-\infty}^{0}\left\{\sum_{j_{1}=1}^{\infty} a_{n+j_{1}+1} \sum_{j_{2}=1}^{\infty} b_{n+j_{2}}^{j_{1}} \cdots \sum_{j_{2 m}=1}^{\infty} b_{n+j_{2 m}}^{j_{2 m-1}} c_{j_{2 m}-p}\right\}^{2} \\
& +\sum_{p=1}^{\infty}\left\{\sum_{j_{1}=1}^{\infty} a_{n+j_{1}+1} \sum_{j_{2}=1}^{\infty} b_{n+j_{2}}^{j_{1}} \cdots \sum_{j_{2 m}=p}^{\infty} b_{n+j_{2 m}}^{j_{2 m-1}} c_{j_{2 m}-p}\right\}^{2} \\
= & \sum_{p=0}^{\infty}\left\{e_{2 m}(n, p)\right\}^{2}+\sum_{p=0}^{\infty}\left\{d_{2 m}(n, p)\right\}^{2},
\end{aligned}
$$

Thus, from Propositions 6.1 and 6.2, it follows that

$$
\begin{aligned}
\sum_{k=1}^{2 m-1} A_{k} \sin ^{2 k}(\pi d) & \leq \liminf _{n \rightarrow \infty} \epsilon(n) n \leq \limsup _{n \rightarrow \infty} \epsilon(n) n \\
& \leq \sum_{k=1}^{2 m} A_{k} \sin ^{2 k}(\pi d)+B_{2 m}(d) \sin ^{4 m}(\pi d) .
\end{aligned}
$$


We are about to estimate the last term. By (6.8), for $k \geq 3$,

$$
\begin{aligned}
B_{k}(d) & \leq \frac{d^{2}}{\pi^{2 k}} \int_{0}^{\infty} d u\left\{\int_{0}^{\infty} \frac{d v_{k}}{\left(v_{k}+u\right)^{1-d}} \int_{0}^{\infty} \frac{d s_{k-1}}{\left(v_{k}+s_{k-1}\right)^{1+d}}\right. \\
& \left.\int_{0}^{\infty} \frac{d s_{k-2}}{\left(s_{k-1}+s_{k-2}\right)} \cdots \int_{0}^{\infty} \frac{d s_{2}}{\left(s_{3}+s_{2}\right)} \int_{0}^{\infty} \frac{d s_{1}}{\left(s_{2}+s_{1}\right)\left(s_{1}+1\right)}\right\}^{2} \\
& =\frac{d^{2}}{\pi^{2 k}}\left\|K_{d} H^{k-2} f\right\|^{2},
\end{aligned}
$$

where $f(x):=1 /(1+x)$, and $H$ is the bounded linear operator on $L^{2}((0, \infty), d u)$ defined by

$$
H g(u):=\int_{0}^{\infty} \frac{1}{u+v} g(v) d v .
$$

Since, by Hilbert's theorem (cf. [HLP, Theorems 316 and 317]), the operator norm $\|H\|$ of $H$ is equal to $\pi$, this inequality and Lemma 6.3 yield

$$
B_{k}(d) \leq \frac{\tan ^{2}(\pi d)}{\pi^{2}} \quad(k \geq 3) .
$$

Thus, if we let $m \rightarrow \infty$ in (6.13), then we obtain

$$
\lim _{n \rightarrow \infty} \epsilon(n) n=\sum_{k=1}^{\infty} A_{k} \sin ^{2 k}(\pi d) .
$$

By Lemma 6.5 below, the right-hand side is equal to $d^{2}$. Thus (6.12) follows.

Lemma 6.5. For $|x|<1, \sum_{k=1}^{\infty} A_{k} x^{2 k}=\pi^{-2} \arcsin ^{2} x$.

Though this is a purely analytic assertion, we give a proof based on results for the $\operatorname{ARIMA}(0, d, 0)$ processes.

Proof. For $0<d<\frac{1}{2}$, let $\left\{Y_{n}: n \in \mathbb{Z}\right\}$ be a $\operatorname{ARIMA}(0, d, 0)$ process such that $E\left[Y_{0}^{2}\right]=\Gamma(1-2 d) / \Gamma^{2}(1-d)$ (see Granger-Joyeux [GJ] and Hosking [Ho]; see also $[\mathrm{BD}, \S 13.2])$. We denote by $\gamma^{\prime}(\cdot),\left\{c_{n}^{\prime}\right\},\left\{a_{n}^{\prime}\right\}, \alpha^{\prime}(\cdot)$, and $\epsilon^{\prime}(\cdot)$ the autocovariance function, sequence of $\mathrm{MA}(\infty)$ coefficients, sequence of $\operatorname{AR}(\infty)$ coefficients, partial autocorrelation function, and function defined by (4.11), respectively, of $\left\{Y_{n}\right\}$. Then we have

$$
\begin{gathered}
a_{n}^{\prime}=-\frac{\Gamma(n-d)}{\Gamma(n+1) \Gamma(-d)}, \quad c_{n}^{\prime}=\frac{\Gamma(n+d)}{\Gamma(n+1) \Gamma(d)} \quad(n \geq 0), \\
\gamma^{\prime}(n) \sim n^{2 d-1} \frac{\Gamma(1-2 d)}{\Gamma(d) \Gamma(1-d)} \quad(n \rightarrow \infty)
\end{gathered}
$$

(see, for example, $[\mathrm{BD}, \S 13.2])$. These imply that $\left\{Y_{n}\right\}$ satisfies $(\mathrm{C} 1),(\mathrm{C} 2),(\mathrm{A} 1)$, and (2.5). Hence it follows from (6.14) that $n \epsilon^{\prime}(n)$ tends to $\sum_{1}^{\infty} A_{k} \sin ^{2 k}(\pi d)$ as 
$n \rightarrow \infty$. On the other hand, by [Ho, Theorem 1] (see also [BD, (13.2.10)]),

$$
\alpha^{\prime}(n)=\frac{d}{n-d} \sim \frac{d}{n} \quad(n \rightarrow \infty)
$$

which, by (6.22) and (6.23) below, implies

$$
\epsilon^{\prime}(n) \sim \sum_{k=n}^{\infty} \alpha^{\prime}(k)^{2} \sim \frac{d^{2}}{n} \quad(n \rightarrow \infty) .
$$

Thus, $\sum_{1}^{\infty} A_{k} \sin ^{2 k}(\pi d)=d^{2}$. The lemma follows if we substitute $\pi^{-1} \arcsin x$ with $0<x<1$ into $d$.

Remark. From Lemma 6.5, it follows that

$$
A_{k}=\frac{1}{\pi^{2}} \cdot \frac{(2 k-2) ! !}{(2 k-1) ! ! k} \quad(k \in \mathbb{N}) .
$$

Next we consider the boundary case.

Theorem 6.6. Let $d=0$, and let $\ell \in \mathcal{R}_{0}$ such that $\int^{\infty} \ell(s) d s / s=\infty$. We assume (C1), (C2), (A1), and (A2). Then (2.5) implies

$$
\epsilon(n) \sim n^{-1}\left\{\frac{\ell(x)}{2 \tilde{\ell}(x)}\right\}^{2} \quad(n \rightarrow \infty) .
$$

Proof. From (4.1) and (4.15), we obtain the lower bound estimate

$$
\begin{aligned}
\epsilon(n) & \geq\left(c_{0}\right)^{-2}\left\|P_{[-n, \infty)}^{\perp} P_{(-\infty, 0]} X_{1}\right\|^{2}=\sum_{p=0}^{\infty}\left\{d_{1}(n, p)\right\}^{2} \\
& =\sum_{p=0}^{\infty}\left(\sum_{v=0}^{\infty} c_{v} a_{v+n+2+p}\right)^{2}=\sum_{p=n+2}^{\infty}\left(\sum_{v=0}^{\infty} c_{v} a_{v+p}\right)^{2} .
\end{aligned}
$$

On the other hand, from (4.1) and the equality

$$
P_{(-\infty, 0]} X_{1}=c_{0} \sum_{v=0}^{\infty} a_{v+1} X_{-v}
$$

we obtain the upper bound estimate

$$
\begin{aligned}
\epsilon(n) & =\left(c_{0}\right)^{-2}\left\|P_{[-n, 0]}^{\perp} P_{(-\infty, 0]} X_{1}\right\|^{2}=\left\|P_{[-n, 0]} \sum_{v=n+1}^{\infty} a_{v+1} X_{-v}\right\|^{2} \\
& \leq\left\|\sum_{v=n+1}^{\infty} a_{v+1} X_{-v}\right\|^{2}=\sum_{v=n+1}^{\infty} \sum_{m=n+1}^{\infty} a_{v+1} a_{m+1} \gamma(m-v) \\
& =\gamma(0) \sum_{m=n+1}^{\infty}\left(a_{m+1}\right)^{2}+2 \sum_{m=n+1}^{\infty} a_{m+1} \sum_{v=1}^{\infty} a_{m+v+1} \gamma(v) .
\end{aligned}
$$

First, we consider the lower bound. By (5.29) and Theorem 5.2,

$$
\sum_{u=0}^{n} c_{u} \sim\{2 \tilde{\ell}(n)\}^{1 / 2} \quad(n \rightarrow \infty)
$$

In particular, $a_{v+p+1} \sum_{u=0}^{v} c_{u} \rightarrow 0$ as $v \rightarrow \infty$. Summing by parts,

$$
\sum_{v=0}^{\infty} c_{v} a_{v+n}=\sum_{v=0}^{\infty}\left(\sum_{35}^{v} c_{u=0}\right)\left(a_{v+n}-a_{v+n+1}\right) .
$$


Now, by (A2), Theorem 5.2, and the monotone density theorem, we have

$$
a_{n}-a_{n+1} \sim n^{-2} \ell(n)\{2 \tilde{\ell}(n)\}^{-3 / 2} \quad(n \rightarrow \infty) .
$$

Thus it follows from [I6, Proposition 4.3] that

$$
\sum_{v=0}^{\infty} c_{v} a_{v+n} \sim n^{-1} \frac{\ell(n)}{2 \tilde{\ell}(n)} \quad(n \rightarrow \infty)
$$

whence

$$
\sum_{p=n+2}^{\infty}\left(\sum_{v=0}^{\infty} c_{v} a_{v+p}\right)^{2} \sim n^{-1}\left\{\frac{\ell(n)}{2 \tilde{\ell}(n)}\right\}^{2} \quad(n \rightarrow \infty) .
$$

Next, we consider the upper bound. As in (6.19), we have

$$
\sum_{v=1}^{\infty} a_{n+v+1} \gamma(v) \sim n^{-1} \frac{\ell(n)}{2^{3 / 2} \tilde{\ell}(n)^{1 / 2}} \quad(n \rightarrow \infty),
$$

whence

$$
2 \sum_{m=n+1}^{\infty} a_{m+1} \sum_{v=1}^{\infty} a_{m+v+1} \gamma(v) \sim n^{-1}\left\{\frac{\ell(n)}{2 \tilde{\ell}(n)}\right\}^{2} \quad(n \rightarrow \infty) .
$$

On the other hand, by Theorem 5.2,

$$
\sum_{m=n+1}^{\infty}\left(a_{m+1}\right)^{2} \sim n^{-1} \ell(n)^{2}\{2 \tilde{\ell}(n)\}^{-3} \quad(n \rightarrow \infty) .
$$

Since $\tilde{\ell}(n) \rightarrow \infty$ as $n \rightarrow \infty$, the latter is negligible in comparison with the former, whence

$$
\gamma(0) \sum_{m=n+1}^{\infty}\left(a_{m+1}\right)^{2}+2 \sum_{m=n+1}^{\infty} a_{m+1} \sum_{v=1}^{\infty} a_{m+v+1} \gamma(v) \sim n^{-1}\left\{\frac{\ell(n)}{2 \tilde{\ell}(n)}\right\}^{2}
$$

as $n \rightarrow \infty$. Thus the theorem follows.

Finally we consider the intermediate-memory processes.

Theorem 6.7. Let $-\infty<d \leq 0$ and $\ell \in \mathcal{R}_{0}$, where $\int^{\infty} \ell(s) d s / s<\infty$ if $d=0$.

We also assume (C1), (C2), and (A1). Then (2.5) implies

$$
\epsilon(n) \sim \frac{n^{4 d-1} \ell(n)^{2}}{(1-4 d)\left\{\sum_{-\infty}^{\infty} \gamma(k)\right\}^{2}} \quad(n \rightarrow \infty) .
$$

Proof. By Theorem 5.3 as well as [I2, Lemma 5.7] and (5.30), we have

$$
\sum_{v=0}^{\infty} c_{v} a_{v+n} \sim a_{n}\left(\sum_{v=0}^{\infty} c_{v}\right) \sim \frac{n^{2 d-1} \ell(n)}{\sum_{-\infty}^{\infty} \gamma(k)} \quad(n \rightarrow \infty),
$$

so that

$$
\sum_{p=n+2}^{\infty}\left(\sum_{v=0}^{\infty} c_{v} a_{v+p}\right)^{2} \sim \frac{n^{4 d-1} \ell(n)^{2}}{(1-4 d)\left\{\sum_{-\infty}^{\infty} \gamma(k)\right\}^{2}} \quad(n \rightarrow \infty)
$$


On the other hand, it follows from Theorem 5.3 and [I2, Lemma 5.7] that

$$
\begin{aligned}
\gamma(0) & \sum_{m=n+1}^{\infty}\left(a_{m+1}\right)^{2}+2 \sum_{m=n+1}^{\infty} a_{m+1} \sum_{v=1}^{\infty} a_{m+v+1} \gamma(v) \\
& \sim\left\{\gamma(0)+2 \sum_{v=1}^{\infty} \gamma(v)\right\} \sum_{m=n+1}^{\infty}\left(a_{m+1}\right)^{2} \quad(n \rightarrow \infty) \\
& \sim \frac{n^{4 d-1} \ell(n)^{2}}{(1-4 d)\left\{\sum_{-\infty}^{\infty} \gamma(k)\right\}^{2}} \quad(n \rightarrow \infty) .
\end{aligned}
$$

Thus the theorem follows from the estimates (6.17) and (6.18).

Now we are ready to prove the main theorem.

Proof of Theorem 2.1. We put

$$
\delta(n):=\frac{\left\|P_{[-n, 0]}^{\perp} X_{1}\right\|^{2}-\left\|P_{[-n-1,0]}^{\perp} X_{1}\right\|^{2}}{\left\|P_{(-\infty, 0]}^{\perp} X_{1}\right\|^{2}} \quad(n=1,2, \ldots) .
$$

Then since $\left\|P_{[-n, 0]}^{\perp} X_{1}\right\| \rightarrow\left\|P_{(-\infty, 0]}^{\perp} X_{1}\right\|$ as $n \rightarrow \infty$, it follows that

$$
\sum_{k=n}^{\infty} \delta(k)=\epsilon(n) \quad(n \geq 1) .
$$

On the other hand, by the Durbin-Levinson algorithm ([BD, (5.2.5)]), we have

$$
\alpha(n)^{2}=\delta(n-2) \frac{\left\|P_{(-\infty, 0]}^{\perp} X_{1}\right\|^{2}}{\left\|P_{[-n+2,0]}^{\perp} X_{1}\right\|^{2}} \sim \delta(n-2) \quad(n \rightarrow \infty) .
$$

Now it follows from (C1)-(A2) and Theorem 4.6 that, for any $k \geq 1$ and $p \geq 0$, both the sequences $\left\{d_{k}(n, p): n=1,2, \ldots\right\}$ and $\left\{d_{k}(n, p)-d_{k}(n+1, p): n=\right.$ $1,2, \ldots\}$ are eventually non-negative and decreasing. Since (4.12) implies

$$
\delta(n)=\sum_{k=1}^{\infty} \sum_{p=0}^{\infty}\left\{d_{k}(n, p)+d_{k}(n+1, p)\right\}\left\{d_{k}(n, p)-d_{k}(n+1, p)\right\},
$$

the sequence $\{\delta(n)\}$ is also eventually decreasing. Hence, by the monotone density theorem, Theorems 6.4, 6.6 and 6.7 give the asymptotics for $\delta(\cdot)$. Therefore, from (6.23), we obtain (2.7)-(2.9). To complete the proof, it suffices to note that (2.6) is equivalent to (2.7), (2.8) or (2.9) under each assumption on $d$ and $\ell(\cdot)$.

\section{ReFleCtion POSITIVITY}

In this section, we consider the stationary processes with reflection positivity, that is, those with completely monotone autocovariance functions. These have the advantage that the relevant series and functions have nice integral representations. In fact, it is shown below that the corresponding sequences $\left\{c_{n}\right\}$ and 
$\left\{a_{n}\right\}$ have such integral representations. These representations in turn imply the conditions (C1)-(A2) immediately, whence we can apply Theorem 2.1 to the stationary processes.

First, we prove some preliminary analytic results. In what follows, we write $\int_{0}^{1}$ for $\int_{[0,1)}$. We put

$$
\Sigma:=\{\sigma: \sigma \text { is a nonzero finite Borel measure on }[0,1)\} .
$$

For $\sigma \in \Sigma$, we write

$$
\Delta_{\sigma}(\theta):=\frac{1}{2 \pi} \int_{0}^{1} P_{r}(\theta) \sigma(d r) \quad(-\pi<\theta<\pi),
$$

where $P_{r}(\theta)$ is the Poisson kernel defined by (3.4). Then the function $\Delta_{\sigma}(\cdot)$ is positive and integrable on $(-\pi, \pi)$. It follows that

$$
\int_{-\pi}^{\pi} e^{i n \theta} \Delta_{\sigma}(\theta) d \theta=\int_{0}^{1} r^{|n|} \sigma(d r) \quad(n \in \mathbb{Z}),
$$

where the convention $0^{0}=1$ is adopted in the integral on the right-hand side.

For a finite Borel measure $\mu$ on $[0,1)$, we write

$$
\begin{aligned}
F_{\mu}(z) & :=\int_{0}^{1} \frac{1}{1-r z} \mu(d r) & (z \in \mathbb{C},|z|<1), \\
F_{\mu}\left(e^{i \theta}\right) & :=\int_{0}^{1} \frac{1}{1-r e^{i \theta}} \mu(d r) & (-\pi<\theta<\pi) .
\end{aligned}
$$

We write $N$ for the set of all nonzero Borel measures $\nu$ on $[0,1)$ such that

$$
\int_{0}^{1} \int_{0}^{1} \frac{1}{1-r s} \nu(d r) \nu(d s)<\infty .
$$

If $\nu \in N$, then $\nu$ is a finite measure, that is, $\nu \in \Sigma$. For $\nu \in N$, we define $\sigma=S(\nu) \in \Sigma$ by

$$
\sigma(d r):=\left\{\int_{0}^{1} \frac{1}{1-r s} \nu(d s)\right\} \nu(d r)
$$

Then we have

$$
\left|F_{\nu}\left(e^{i \theta}\right)\right|^{2}=2 \pi \Delta_{\sigma}(\theta) \quad(-\pi<\theta<\pi)
$$

for

$$
\frac{1-(r+s) \cos \theta+r s}{\left|1-r e^{i \theta}\right|^{2} \cdot\left|1-s e^{i \theta}\right|^{2}}=\frac{1}{2(1-r s)}\left\{P_{r}(\theta)+P_{s}(\theta)\right\} .
$$

In particular, $\int_{-\pi}^{\pi}\left|F_{\nu}\left(e^{i \theta}\right)\right|^{2} d \theta=2 \pi \sigma([0,1))$, whence $F_{\nu}\left(e^{i \theta}\right) \in L^{2}(-\pi, \pi)$ if $\nu \in$ $N$. Moreover, since the real part of $F_{\nu}(z)$ is positive in $|z|<1, F_{\nu}(z)$ is an outer 
function for the space $H^{2+}$, and so $\log \left|F_{\nu}\left(e^{i \theta}\right)\right|$ is integrable on $(-\pi, \pi)$ and

$$
F_{\nu}(z)=\exp \left\{\frac{1}{2 \pi} \int_{-\pi}^{\pi} \frac{e^{i \theta}+z}{e^{i \theta}-z} \log \left|F_{\nu}\left(e^{i \theta}\right)\right| d \theta\right\} \quad(|z|<1)
$$

(see Duren [Du, Chapter 3, Exercise 1] and [Ru, Theorem 17.16]). If $\sigma=S(\nu)$, then, from (7.3) and (7.4), it follows that $\log \Delta(\cdot)$ is also integrable on $(-\pi, \pi)$ and that

$$
F_{\nu}(z)=(2 \pi)^{1 / 2} \exp \left\{\frac{1}{4 \pi} \int_{-\pi}^{\pi} \frac{e^{i \theta}+z}{e^{i \theta}-z} \log \left|\Delta_{\sigma}(\theta)\right| d \theta\right\} \quad(z \in \mathbb{C},|z|<1) .
$$

The following theorem is a discrete-time analogue of [I3, Theorem 2.5].

Theorem 7.1. The map $S$ from $N$ to $\Sigma$ is one-to-one and onto.

Proof. Step 1. For brevity, call a measure $\sigma$ on $[0,1)$ simple if $\sigma$ is of the form

$$
\sigma=\sum_{k=1}^{n} s_{k} \delta_{r_{k}}
$$

for some $n \in \mathbb{N}$, where $s_{k} \in(0, \infty)(k=1, \cdots, n)$ and $0<r_{1}<r_{2}<\cdots<r_{n}<$ 1. In this step, we show that for $\sigma$ simple there exists a simple measure $\nu$ such that $\sigma=S(\nu)$.

For a simple measure $\sigma$ of the above form, we define a polynomial $f(z)$ of degree $2 n-2$ by

$$
f(z):=\sum_{k=1}^{n}\left\{1-\left(r_{k}\right)^{2}\right\} s_{k} \prod_{m \neq k}\left(1-r_{m} z\right)\left(z-r_{m}\right) .
$$

Then, since $f\left(r_{k}\right) f\left(r_{k+1}\right)<0(k=1, \ldots, n-1), f(z)$ has a zero $q_{k}$ in $\left(r_{k}, r_{k+1}\right)$ for $k=1, \ldots, n-1$. Moreover, since $f(1 / z)=z^{-2 n+2} f(z)$, we see that $1 / q_{k}(k=$ $1, \ldots, n-1)$ are also zeros of $f(z)$. Thus $f(z)$ must be of the form

$$
f(z)=c \prod_{k=1}^{n-1}\left(1-q_{k} z\right)\left(z-q_{k}\right)
$$

with some positive constant $c$. Now we define a rational function $F(z)$ by

$$
F(z):=\sqrt{c} \frac{\prod_{k=1}^{n-1}\left(1-q_{k} z\right)}{\prod_{k=1}^{n}\left(1-r_{k} z\right)}
$$

Then

$$
F(z) F(1 / z)=c z \frac{\prod_{k=1}^{n-1}\left(1-q_{k} z\right)\left(z-q_{k}\right)}{\prod_{k=1}^{n}\left(1-r_{k} z\right)\left(z-r_{k}\right)}=\sum_{k=1}^{n} \frac{\left\{1-\left(r_{k}\right)^{2}\right\} s_{k}}{\left(1-r_{k} z\right)\left(1-r_{k} z^{-1}\right)}
$$

so that

$$
\left|F\left(e^{i \theta}\right)\right|^{2}=\lim _{t \rightarrow 1-} F\left(t e^{i \theta}\right) F\left(1 / t e^{i \theta}\right)=2 \pi \Delta_{\sigma}(\theta) .
$$


On the other hand, $F(z)$ has the following partial fraction decomposition:

$$
F(z)=\sum_{k=1}^{u} \frac{m_{k}}{1-r_{k} z}
$$

where $m_{k} \in(0, \infty)(k=1, \ldots, n)$. If we write $\nu:=\sum_{k=1}^{n} m_{k} \delta_{r_{k}}$, then we have $F(z)=F_{\nu}(z)$; and so, by (7.3) and (7.6), $\Delta_{\sigma}(\theta)=\Delta_{\sigma^{\prime}}(\theta)$ with $\sigma^{\prime}:=S(\nu)$. By (7.1), this implies that $\int_{0}^{1} t^{n} \sigma(d t)=\int_{0}^{1} t^{n} \sigma^{\prime}(d t)$ for all $n \in \mathbb{N} \cup\{0\}$. Thus $\sigma=\sigma^{\prime}=S(\nu)$.

Step 2. For $\sigma \in \Sigma$, choose a sequence of simple measures $\sigma_{n}$ such that $\sigma_{n} \rightarrow \sigma$ weakly on $[0,1]$ as $n \rightarrow \infty$. Here we regard $\sigma$ and $\sigma_{n}$ as measures on $[0,1]$ by $\sigma(\{1\})=\sigma_{n}(\{1\})=0$. In view of Step 1 , we have simple measures $\nu_{n}$ such that $S\left(\nu_{n}\right)=\sigma_{n}$. From (7.1), (7.5), and Jensen's inequality, it follows that

$$
\nu_{n}([0,1))=F_{\nu}(0)=(2 \pi)^{1 / 2} \exp \left\{\frac{1}{4 \pi} \int_{-\pi}^{\pi} \log \left|\Delta_{\sigma_{n}}(\theta)\right| d \theta\right\} \leq \sqrt{2 \pi \sigma_{n}([0,1))},
$$

whence, by the Helly selection principle, there exists a sequence of integers $n^{\prime} \rightarrow$ $\infty$ such that $\nu_{n^{\prime}}$ converges weakly on $[0,1]$ to a finite measure, $\nu$. From $(7.3)$, we have

$$
\left|\int_{0}^{1} \frac{1}{1-r e^{i \theta}} \nu_{n^{\prime}}(d r)\right|^{2}=\int_{0}^{1} P_{r}(\theta) \sigma_{n^{\prime}}(d r) \quad(-\pi<\theta<\pi) .
$$

Let $n^{\prime} \rightarrow \infty$. Then since, for $\theta \neq 0$, both integrands are bounded and continuous on $[0,1]$, we have

$$
\left|\int_{[0,1]} \frac{1}{1-r e^{i \theta}} \nu(d r)\right|^{2}=2 \pi \Delta_{\sigma}(\theta) \quad(-\pi<\theta<\pi, \theta \neq 0) .
$$

The absolute value of the integral on the left-hand side is at least

$$
\left|\operatorname{Im} \int_{[0,1]} \frac{1}{1-r e^{i \theta}} \nu(d r)\right| \geq \frac{|\sin \theta|}{2(1-\cos \theta)} \nu(\{1\}) .
$$

Since $\Delta_{\sigma} \in L^{1}(-\pi, \pi)$, this implies that $\nu(\{1\})=0$. We put

$$
\sigma^{\prime}(d r):=\left\{\int_{0}^{1} \frac{1}{1-r s} \nu(d s)\right\} \nu(d r) .
$$

Then

$$
\Delta_{\sigma}(\theta)=\frac{1}{2 \pi} \int_{0}^{1} P_{r}(\theta) \sigma^{\prime}(d r)
$$

and so, as in Step 1, $\int_{0}^{1} t^{n} \sigma(d t)=\int_{0}^{1} t^{n} \sigma^{\prime}(d t)$ for all $n \geq 0$. Consequently, $\nu$ is in $N$, and $\sigma=\sigma^{\prime}=S(\nu)$. Thus $S$ is onto. 
It remains to show that $S$ is one-to-one. By (7.5), we find that $F_{\nu}$ is determined uniquely by $\sigma$. Since $F_{\nu}(z)$ determines $\nu$ uniquely, this implies that $S$ is one-toone.

Theorem 7.2. For every $\nu \in N$, there exists a unique triple $\left(b_{1}, b_{2}, \rho\right)$ consisting of $b_{1} \in(0, \infty), b_{2} \in[0, \infty)$ and a finite (possibly zero) Borel measure $\rho$ on $[0,1)$ such that

$$
F_{\nu}(z)\left\{b_{1}(1-z)+b_{2}(1+z)+\left(1-z^{2}\right) F_{\rho}(z)\right\}=1 \quad(z \in \mathbb{C},|z|<1)
$$

Proof. Let $z \downarrow-1$ or $z \uparrow 1$ in (7.7). Then we obtain

$$
b_{1}=\left\{\int_{0}^{1} \frac{1}{1+r} \nu(d r)\right\}^{-1}, \quad b_{2}=\left\{\int_{0}^{1} \frac{1}{1-r} \nu(d r)\right\}^{-1}
$$

$\left(b_{2}=0\right.$ if $\left.\int_{0}^{1}(1-r)^{-1} \nu(d r)=\infty\right)$. Therefore both $b_{1}$ and $b_{2}$ are uniquely determined by $\nu$. Since $\rho$ is uniquely determined by $F_{\rho}$, it is also uniquely determined by $\nu$.

Now we show the existence. First we assume $\int_{0}^{1}(1-r)^{-1} \nu(d r)<\infty$. Then by [I2, Theorem 3.1(i)], there exist positive constants $\alpha_{2}, \beta_{2}$, and a finite Borel measure $\rho_{2}$ on $[0,1)$ such that $\int_{0}^{1}(1+r)^{-1} \rho_{2}(d r)<1$ and

$$
\frac{\alpha_{2}}{\sqrt{2 \pi}}\left\{\frac{\beta_{2}}{2}(1+z)+(1-z)+z(1-z) F_{\rho_{2}}(z)\right\} F_{\nu}(z)=1 \quad(z \in \mathbb{C},|z|<1) .
$$

Since

$$
z F_{\rho_{2}}(z)=-\int_{0}^{1} \frac{1}{1+r} \rho_{2}(d r)+(1+z) \int_{0}^{1} \frac{1}{(1-r z)(1+r)} \rho_{2}(d r)
$$

it follows that (7.7) holds with

$$
b_{1}=\frac{\alpha_{2}}{\sqrt{2 \pi}}\left\{1-\int_{0}^{1} \frac{1}{1+r} \rho_{2}(d r)\right\}, \quad b_{2}=\frac{\alpha_{2} \beta_{2}}{2 \sqrt{2 \pi}}, \quad \rho(d r)=\frac{\alpha_{2}}{\sqrt{2 \pi}(1+r)} \rho_{2}(d r)
$$

Next we assume $\int_{0}^{1}(1-r)^{-1} \nu(d r)=\infty$. We put

$$
\nu^{(n)}(d r):=I_{\left[0,1-n^{-1}\right]}(r) \nu(d r) \quad(n \geq M)
$$

where we choose $M$ so large that $\nu^{(M)}$ is not a zero measure. By the result above, there exist $b_{1}^{(n)} \in(0, \infty), b_{2}^{(n)} \in(0, \infty)$, and a finite Borel measure $\rho^{(n)}$ on $[0,1)$ 
which satisfy (7.7) with $\nu^{(n)}$. By (7.8),

$$
\begin{aligned}
& b_{1}^{(n)}=\left\{\int_{0}^{1} \frac{1}{1+r} \nu^{(n)}(d r)\right\}^{-1} \quad \downarrow \quad b_{1}:=\left\{\int_{0}^{1} \frac{1}{1+r} \nu(d r)\right\}^{-1} \quad(n \rightarrow \infty), \\
& b_{2}^{(n)}=\left\{\int_{0}^{1} \frac{1}{1-r} \nu^{(n)}(d r)\right\}^{-1} \quad \downarrow \quad 0 \quad(n \rightarrow \infty) .
\end{aligned}
$$

On the other hand, if we let $z=0$ in (7.7), then we obtain

$$
\sup _{n \geq M} \rho^{(n)}([0,1)) \leq \sup _{n \geq M} \frac{1}{\nu^{(n)}([0,1))}<\infty .
$$

Thus, by a standard argument which involves the Helly selection principle, there exists a finite Borel measure $\rho$ on $[0,1)$ such that the triple $\left(b_{1}, 0, \rho\right)$ satisfies $(7.7)$ with $\nu$.

Let us come back to stationary processes. For $\sigma \in \Sigma$, the function $\gamma(\cdot)$ defined by $\gamma(n):=\int_{0}^{1} t^{|n|} \sigma(d t)(n \in \mathbb{Z})$ is non-negative definite by (7.1), whence it is the autocovariance function of a stationary process with spectral density $\Delta_{\sigma}$. A stationary process $\left\{X_{n}\right\}$ with autocovariance function $\gamma(\cdot)$ has the property of reflection positivity if

(RP) there exists $\sigma \in \Sigma$ such that $\gamma(n)=\int_{0}^{1} t^{|n|} \sigma(d t) \quad(n \in \mathbb{Z})$.

Since $\log \Delta_{\sigma}(\cdot)$ is integrable on $(-\pi, \pi)$ for $\sigma \in \Sigma$, it follows that such a stationary process is purely nondeterministic.

Now we prove that (RP) implies (C1)-(A2).

Theorem 7.3. Let $\left\{X_{n}\right\}$ be a stationary process that satisfies (RP). Then there exist two finite Borel measures $\nu$ and $\rho$ on $[0,1)$ such that

$$
\begin{gathered}
c_{n}=\int_{0}^{1} r^{n} \nu(d r) \quad(n=0,1, \ldots), \\
a_{n}=\int_{0}^{1} r^{n-2}\left(1-r^{2}\right) \rho(d r) \quad(n=2,3, \ldots) .
\end{gathered}
$$

In particular, $\left\{X_{n}\right\}$ satisfies $(\mathrm{C} 1),(\mathrm{C} 2),(\mathrm{A} 1)$, and $(\mathrm{A} 2)$.

Proof. Let $\sigma$ be the measure that appears in (RP). Set $\nu:=S^{-1}(\sigma) \in N$. Then, by (7.5), the outer function $h(z)$ of $\left\{X_{n}\right\}$ is equal to $F_{\nu}(z)$. Therefore it follows that the $\mathrm{MA}(\infty)$ coefficients $c_{n}$ of $\left\{X_{n}\right\}$ are given by (7.9) with this $\nu$. Let $\left(b_{1}, b_{2}, \rho\right)$ be the triple determined by the relation (7.7). Then, for $z \in \mathbb{C}$,

$$
-1 / h(z)=-1 / F_{\nu}(z)=-b_{1}(1-z)-b_{2}(1+z)-\left(1-z^{2}\right) F_{\rho}(z) .
$$


Thus (7.10) holds with this $\rho$.

Example. Let $-\infty<d<\frac{1}{2}$, and let $\left\{X_{n}\right\}$ be a stationary process with autocovariance function of the form $\gamma(n)=(1+|n|)^{-(1-2 d)}$. Since

$$
\frac{1}{(1+|n|)^{1-2 d}}=\int_{0}^{1} t^{|n|} \frac{(-\log t)^{-2 d}}{\Gamma(1-2 d)} d t \quad(n \in \mathbb{Z}),
$$

we see that $\left\{X_{n}\right\}$ satisfies $(\mathrm{RP})$. Let $\alpha(\cdot)$ be the partial autocorrelation function of $\left\{X_{n}\right\}$. Then application of Theorem 2.1 to $\left\{X_{n}\right\}$ yields the following result:

(1) if $0<d<\frac{1}{2}$, then

$$
|\alpha(n)| \sim \frac{d}{n} \quad(n \rightarrow \infty)
$$

(2) if $d=0$, then

$$
|\alpha(n)| \sim \frac{1}{2 n \log n} \quad(n \rightarrow \infty)
$$

(3) if $-\infty<d<0$, then

$$
|\alpha(n)| \sim \frac{n^{2 d-1}}{\{2 \zeta(1-2 d)-1\}} \quad(n \rightarrow \infty) .
$$

Here $\zeta(s)$ is the Riemann zeta function.

In view of the numerical data obtained using the Durbin-Levinson algorithm, it seems unnecessary to take the absolute values of $\alpha(n)$ in (1)-(3) above. This observation even suggests a possible improvement of Theorem 2.1.

We have seen that the stationary processes with reflection positivity satisfy the conditions (C1)-(A2). We must, however, point out that it would be desirable to remove the assumption (C1) from Theorem 2.1.

Acknowledgement. I should like to thank Professor Takahiko Nakazi for helpful comments on the Seghier-Dym theorem.

\section{REFERENCES}

[BGT] N. H. Bingham, C. M. Goldie, and J. L. Teugels, Regular variation, 2nd edn, Cambridge University Press, 1989; 1st edn 1987.

[BD] P. J. Brockwell and R. A. Davis, Time series : Theory and methods, 2nd edn, SpringerVerlag, New York, 1991.

[Du] P. L. Duren, Theory of $H^{p}$ spaces, Academic Press, New York, 1970.

[Dy1] H. Dym, Trace formulas for a class of Toeplitz-like operators, Israel J. Math. 27 (1977), $21-48$. 
[Dy2] H. Dym, A problem in trigonometric approximation theory, Illinois J. Math. 22 (1978), 402-403.

[DM] H. Dym and H. P. McKean, Gaussian processes, function theory, and the inverse spectral problem, Academic Press, New York, 1976.

[GJ] C. W. Granger and R. Joyeux, An introduction to long-memory time series models and fractional differencing, J. Time Series Analysis 1 (1980), 15-29.

[HLP] G. H. Hardy, J. E. Littlewood, and G. Pólya, Inequalities, 2nd edn, Cambridge University Press, 1952.

[Ha] P. R. Halmos, A Hilbert space problem book, 2nd edn, Springer-Verlag, New York, 1982.

[Ho] J. R. M. Hosking, Fractional differencing, Biometrika 68 (1981), 165-176.

[IR] I. A. Ibragimov and Y. A. Rozanov, Gaussian random processes, Springer-Verlag, New York, 1978.

[I1] A. Inoue, The Alder-Wainwright effect for stationary processes with reflection positivity, J. Math. Soc. Japan 43 (1991), 515-526.

[I2] A. Inoue, The Alder-Wainwright effect for stationary processes with reflection positivity (II), Osaka J. Math. 28 (1991), 537-561.

[I3] A. Inoue, On the equations of stationary processes with divergent diffusion coefficients, J. Fac. Sci. Univ. Tokyo Sec. IA 40 (1993), 307-336.

[I4] A. Inoue, On Abel-Tauber theorems for Fourier cosine transforms, J. Math. Anal. Appl. 196 (1995), 764-776.

[I5] A. Inoue, Abel-Tauber theorems for Fourier-Stieltjes coefficients, J. Math. Anal. Appl. 211 (1997), 460-480.

[I6] A. Inoue, Regularly varying correlation functions and KMO-Langevin equations, Hokkaido Math. J. 26 (1997), 1-26.

[IK] A. Inoue and Y. Kasahara, On the asymptotic behavior of the prediction error of a stationary process, in Trends in Probability and Related Analysis (N. Kono and N. R. Shieh, eds.), World Scientific, Singapore, 1999, pp. 207-218.

[LM] N. Levinson and H. P. McKean, Weighted trigonometrical approximation on $R^{1}$ with application to the germ field of a stationary Gaussian noise, Acta Math. 112 (1964), 98-143.

[O] Y. Okabe, On the theory of discrete KMO-Langevin equations with reflection positivity (I), Hokkaido Math. J. 16 (1987), 315-341.

[OI] Y. Okabe and A. Inoue, On the exponential decay of the correlation functions for KMO-Langevin equations, Japan. J. Math. 18 (1992), 13-24.

[Ro] Y. A. Rozanov, Stationary random processes, Holden-Day, San Francisco, 1967.

[Ru] W. Rudin, Real and complex analysis, 3rd edn, McGraw-Hill, New York, 1987.

[S] A. Seghier, Prediction d'un processus stationnaire du second ordre de covariance connue sur un intervalle fini, Illinois J. Math. 22 (1978), 389-401.

[Z] A. Zygmund, Trigonometric series, 2nd edn, Cambridge University Press, 1959.

Department of Mathematics, Faculty of Science, Hokkaido University, SapPORO 060, JAPAN

E-mail address: inoue@math.sci.hokudai.ac.jp 